\title{
One-dimensional nuclear dynamics in the time-dependent Hartree-Fock approximation*
}

\author{
P. Bonche, ${ }^{\dagger}$ S. Koonin, ${ }^{\ddagger}$ and J. W. Negele ${ }^{\S}$ \\ Laboratory for Nuclear Science and Department of Physics, Massachusetts Institute of Technology, Cambridge, Massachusetts 02139 \\ (Received 14 October 1975)
}

\begin{abstract}
The time-dependent Hartree-Fock theory is applied to the large amplitude dynamics of slabs of spin and isospin symmetric nuclear matter. The slabs are translationally invariant in two transverse dimensions, and with the simplified effective interaction used in this work, the problem is reduced to a set of coupled nonlinear equations for time-dependent functions of a single spatial variable. By specification of appropriate initial conditions, large amplitude oscillations of a single slab, the scattering of a slab from an external potential barrier, and collisions of two slabs have been investigated. The results evidence a wide variety of dynamic phenomena, including fusion, compound nucleus formation, dissipation, strongly damped collisions, shock wave propagation, and fragmentation. The microscopic aspects of the dynamics, the relation to fluid mechanics, and the practical and conceptual problems arising from the theory are discussed in detail.
\end{abstract}

NUCLEAR STRUCTURE Time-dependent Hartree-Fock approximation applied to nuclear dynamics. Describes large amplitude collective motion, collisions, dissipation, shock propagation.

\section{INTRODUCTION}

A fundamental understanding of the dynamics of finite nuclei requires a suitable approximation to the time-dependent quantal many-body wave function. Given the two-body interaction and appropriate initial conditions, this approximation should allow the system itself to select the relevant collective or single-particle degrees of freedom, without their being imposed by deliberate ansatz. From such an approximation, one would hope to obtain a unified microscopic description of a wide range of dynamic phenomena, including large amplitude collective oscillations, fission, fusion, compound nucleus formation, dissipation, shock wave propagation, and fragmentation. In addition, it would enable one to assess the validity of the adiabatic assumption frequently invoked in studies of collective motion, and it could provide substantial insight into the possible connection between the quantal wave function and the variables of fluid dynamics.

This work discusses the implementation and applicability of such an approximation, the timedependent Hartree-Fock (TDHF) approximation. First proposed by Dirac ${ }^{1}$ in 1930, TDHF describes the many-body wave function by a single Slater determinant. This determinant is specified by the criterion that at every time, the deviation between it and the solution to the Schrödinger equation is minimized, or equivalently, that $\langle\psi|(i \hbar \partial / \partial t-H)| \psi\rangle$ is stationary when $\psi$ is restricted to the space of single determinants. The single-particle wave functions comprising $\psi$ provide an optimal timedependent basis for describing the full many-body wave function in the sense that all one-particle one-hole admixtures are identically included, and the first nonvanishing correction terms are twoparticle two-hole states. This basis is quite distinct from the instantaneous static basis obtained by filling the lowest available singleparticle states in the Hartree-Fock (HF) potential generated by the density at any specific time, and thus TDHF contains essential physics which must be described in terms of very complicated excitations in the instantaneous static basis.

The choice of the TDHF approximation is motivatived by several considerations. Two limiting cases of TDHF, when used with appropriate effective interactions, provide excellent descriptions of appropriate nuclear properties. The static HF approximation accurately predicts energies and charge density distributions in spherical and welldeformed nuclei throughout the Periodic Table. ${ }^{2-5}$ In the small-amplitude limit, TDHF reduces to the random phase approximation (RPA) which quantitatively describes the excitation energies and transition charge densities associated with appropriate low lying collective states. ${ }^{6}$ In addition, the mean-field aspect of TDHF is intuitively appealing in the sense that the mean field is the obvious candidate to communicate collective information in large amplitude dynamics. The time reversal property of TDHF is another advantage of this approximation. Although it allows the free exchange of excitation energy between collective and intrinsic degrees of freedom, which is often described in terms of viscosity or dissipation, the theory is invariant under time reversal thus en- 
suring microreversibility of reactions. Finally, such a time-dependent variational theory offers the hope of beginning to develop a systematic approach to the general problem of the dynamics of composite systems which, for example, should ultimately be relevant to understanding the dynamics of hadrons. ${ }^{7}$

The organization of this paper is as follows. The general TDHF formalism is reviewed in Sec. II. For the purposes of this present exploratory investigation, a greatly simplified effective interaction has been developed which is described in Sec. III. In order to limit the computational scope of this work, we have restricted our attention to the dynamics of slabs of spin and isospin symmetric nuclear matter. Section IV describes the specialization to such slab geometry and presents the HF results for static slabs. In Sec. V, we discuss the specification of initial conditions and explore the dynamics of a single slab. Our primary application of TDHF has been to the collisions of two slabs. The resulting collision phenomenology is presented in Sec. VI and includes inelastic scattering, compound nucleus formation, resonance behavior, strongly damped collisions with associated dissipation, mass transfer, fragmentation, and shock propagation. In order to gain insight into the underlying structure of the approximation, Sec. VII explores several microscopic aspects, including the role of single-particle effects and the off-diagonal behavior of the one-body density matrix. Finally, in Sec. VIII, we emphasize some of the practical and conceptual problems arising from this investigation and discuss possibilities for future work which appear promising.

\section{GENERAL FORMALISM}

In this section, we briefly review the TDHF equations and cast them into a form suitable for numerical calculations. For the special case of a Yukawa two-body force with density-dependent terms in a spin-isospin saturated system, we give explicit expressions for the HF potential.

\section{TDHF equations}

The full many-body wave function for $A$ nucleons $\Psi$ satisfies the time-dependent Schrödinger equation

$$
i \hbar \dot{\Psi}=\hat{H} \Psi,
$$

where the dot denotes time differentiation. The Hamiltonian operator $\hat{H}$ is given by

$$
\hat{H}=\sum_{i=1}^{A} \hat{K}_{i}+\frac{1}{2} \sum_{i \neq j=1}^{A} \hat{V}_{i j},
$$

where $\hat{K}$ is the one-body kinetic energy operator and $\hat{V}$ the two-body potential.

The TDHF approximation consists of assuming that the full wave function $\Psi$ is a single time-dependent Slater determinant ${ }^{8}$ :

$\Psi\left(\overrightarrow{\mathrm{r}}_{1}, \overrightarrow{\mathrm{r}}_{2}, \ldots, \overrightarrow{\mathrm{r}}_{A}\right) \simeq \hat{\mathrm{Q}}\left[\psi_{1}\left(\overrightarrow{\mathrm{r}}_{1}, t\right) \psi_{2}\left(\overrightarrow{\mathrm{r}}_{2}, t\right) \cdots \psi_{A}\left(\overrightarrow{\mathrm{r}}_{A}, t\right)\right]$,

where the right-hand side is an antisymmetrized product of $A$ complex single-particle wave functions $\psi_{i}$. The coordinates $\overrightarrow{\mathbf{r}}_{i}$ include spin and isospin labels. The $\psi_{i}$ 's are chosen or thonormal at some initial time $t_{0}$, which insures the normalization of $\Psi$. As shown below, they will retain this property at all other times, i.e.,

$$
\int d \overrightarrow{\mathbf{r}} \psi_{i}^{*}(\overrightarrow{\mathbf{r}}, t) \psi_{j}(\overrightarrow{\mathbf{r}}, t)=\delta_{i j} .
$$

The TDHF equations for the single-particle wave functions are

$$
i \hbar \dot{\psi}_{i}(\overrightarrow{\mathbf{r}}, t)=\int h\left(\overrightarrow{\mathbf{r}}, \overrightarrow{\mathbf{r}}^{\prime}, t\right) \psi_{i}\left(\overrightarrow{\mathbf{r}}^{\prime}, t\right) d \overrightarrow{\mathbf{r}}^{\prime} .
$$

The nonlocal HF Hamiltonian is given by

$$
h\left(\overrightarrow{\mathrm{r}}, \overrightarrow{\mathrm{r}}^{\prime}, t\right)=K\left(\overrightarrow{\mathrm{r}}, \overrightarrow{\mathrm{r}}^{\prime}\right)+W\left(\overrightarrow{\mathrm{r}}, \overrightarrow{\mathrm{r}}^{\prime}, t\right) .
$$

The kinetic energy operator is defined as

$$
K\left(\overrightarrow{\mathrm{r}}, \overrightarrow{\mathbf{r}}^{\prime}\right)=-\frac{\hbar^{2}}{2 m} \nabla^{2} \delta\left(\overrightarrow{\mathrm{r}}-\overrightarrow{\mathrm{r}}^{\prime}\right),
$$

where $m$ is the nucleon mass. The HF potential is given by

$$
\begin{aligned}
W\left(\overrightarrow{\mathbf{r}}, \overrightarrow{\mathbf{r}}^{\prime}, t\right)=\sum_{n} \int & \tilde{V}\left(\overrightarrow{\mathbf{r}}, \overrightarrow{\mathbf{r}}^{\prime \prime}, \overrightarrow{\mathbf{r}}^{\prime}, \overrightarrow{\mathbf{r}}^{\prime \prime \prime}\right) \\
& \times \psi_{n}^{*}\left(\overrightarrow{\mathbf{r}}^{\prime \prime}, t\right) \psi_{n}\left(\overrightarrow{\mathbf{r}}^{\prime \prime \prime}, t\right) d \overrightarrow{\mathbf{r}}^{\prime \prime} d \overrightarrow{\mathbf{r}}^{\prime \prime \prime},
\end{aligned}
$$

where $\tilde{V}$ is the antisymmetrized two-body potential. Equation (2.5) may be rewritten as

$$
\begin{aligned}
i \hbar \dot{\psi}_{i}(\overrightarrow{\mathrm{r}}, t)= & -\frac{\hbar^{2}}{2 m} \nabla^{2} \psi_{i}(\overrightarrow{\mathrm{r}}, t) \\
& +\int W\left(\overrightarrow{\mathbf{r}}, \overrightarrow{\mathbf{r}}^{\prime}, t\right) \psi_{i}\left(\overrightarrow{\mathbf{r}}^{\prime}, t\right) d r^{\prime} .
\end{aligned}
$$

This expression is a one-body Schrödinger equation for $\psi_{i}$ with a nonlocal potential $W$ determined by the instantaneous wave functions of all the other particles. The TDHF approximation thus reduces the many-body problem [cf. Eq. (2.1)] to a set of $A$ coupled nonl inear integrodifferential equations for the single-particle wave functions $\psi_{i}$. Therefore, numerical solution of the TDHF equations is no more difficult than the solution of $A$ coupled one-body equations. 
At this point, it is worthwhile to note that the coupled set of Eqs. (2.9) is only a particular representation of the TDHF equations. Indeed, TDHF has often been expressed as $^{8,9}$

$$
i \hbar \dot{\rho}=[h, \rho],
$$

where $\rho$ is the one-body density matrix

$$
\rho\left(\overrightarrow{\mathrm{r}}, \overrightarrow{\mathbf{r}}^{\prime}, t\right)=\sum_{i=1}^{A} \psi_{i}(\overrightarrow{\mathrm{r}}, t) \psi_{i}^{*}\left(\overrightarrow{\mathrm{r}}^{\prime}, t\right) .
$$

A numerical solution of Eq. (2.10) would evolve in time the density matrix defined at discrete values of both $\overrightarrow{\mathbf{r}}$ and $\overrightarrow{\mathbf{r}}^{\prime}$. On the other hand, the Eqs. (2.9) treat $A$ functions $\left\{\psi_{i}\right\}$ of a single coordinate. Insofar as the number of single-particle wave functions is generally much smaller than the number of discrete values used for any single coordinate, it is far more efficient to evolve the singleparticle wave functions through the Set (2.9). In addition, if need be, the one-body density matrix can always be constructed very easily from the single-particle wave functions via Eq. (2.11).

\section{Conservation laws}

Several quantities are conserved by the TDHF equations. As mentioned above, the scalar product of two single-particle wave functions remains constant in time. From Eq. (2.5), each $\psi_{i}$ evolves in time with the same Hermitian effective Hamiltonian $h$, so that

$$
\frac{d}{d t} \int \psi_{i}^{*}(\overrightarrow{\mathbf{r}}, t) \psi_{j}(\overrightarrow{\mathbf{r}}, t) d \overrightarrow{\mathbf{r}}=0 .
$$

Thus, the metric of the $\left\{\psi_{i}\right\}$ is time-independent. From Eq. (2.10) it follows that the number of nucleons, given by

$$
A=\sum_{i=1}^{A} \int \psi_{i}^{*}(\overrightarrow{\mathrm{r}}, t) \psi_{i}(\overrightarrow{\mathrm{r}}, t) d \overrightarrow{\mathbf{r}}
$$

is also time-independent.

The second conserved quantity is the expectation value of $\hat{H}$, given by

$$
\begin{gathered}
\langle\hat{H}\rangle=E=\sum_{i} \iint \psi_{i}^{*}(\overrightarrow{\mathbf{r}}, t)\left[h\left(\overrightarrow{\mathbf{r}}, \overrightarrow{\mathbf{r}}^{\prime}, t\right)-\frac{1}{2} W\left(\overrightarrow{\mathbf{r}}, \overrightarrow{\mathbf{r}}^{\prime}, t\right)\right] \\
\times \psi_{i}\left(\overrightarrow{\mathbf{r}}^{\prime}, t\right) d \overrightarrow{\mathbf{r}} d \overrightarrow{\mathbf{r}}^{\prime}
\end{gathered}
$$

and may easily be shown to remain a constant in time if the $\psi_{i}$ 's are evolved according to Eq. (2.9).

\section{Single - particle field}

Our present application deals with spin-saturated isoscalar systems. In such cases, the HF potential is independent of both spin and isospin provided the two-body force conserves angular mo- mentum and isospin. The motivation for the choice of the two-body interaction discussed below will be treated in detail in Sec. III. Significant simplification arises from the use of an interaction which yields a local HF potential

$$
W\left(\overrightarrow{\mathbf{r}}, \overrightarrow{\mathbf{r}}^{\prime}\right)=W(\overrightarrow{\mathbf{r}}) \delta\left(\overrightarrow{\mathbf{r}}-\overrightarrow{\mathbf{r}}^{\prime}\right) \text {. }
$$

The force consists of constant and density-dependent $\delta$-function interactions and a direct Yukawa potential. (Alternatively the density-dependent component of the force may be interpreted as a zero-range three-body interaction of strength $t_{3}$ ). The following form for $W$ is then obtained:

$$
\begin{aligned}
W(\overrightarrow{\mathbf{r}})= & \frac{3}{4} t_{0} \rho(\overrightarrow{\mathbf{r}})+\frac{3}{16} t_{3} \rho^{2}(\overrightarrow{\mathbf{r}}) \\
& +V_{0} \int \rho\left(\overrightarrow{\mathbf{r}}^{\prime}\right) \frac{e^{-\left|\overrightarrow{\mathbf{r}}-\overrightarrow{\mathbf{r}}^{\prime}\right| / a}}{\left|\overrightarrow{\mathbf{r}}-\overrightarrow{\mathbf{r}}^{\prime}\right| / a} d \overrightarrow{\mathbf{r}}^{\prime} .
\end{aligned}
$$

In this expression, the nucleon density $\rho$ is given by

$$
\rho(\overrightarrow{\mathrm{r}}, t)=4 \sum_{m=1}^{\frac{1}{4} A}\left|\psi_{m}(\overrightarrow{\mathbf{r}}, t)\right|^{2},
$$

where the $\psi_{m}(\overrightarrow{\mathbf{r}}, t)$ are the time-dependent spatial wave functions, each occupied by four nucleons in different spin and isospin states. We have omitted the Coulomb force, as a consistent treatment for slab geometry would result in an infinite potential.

With $W$ defined according to Eq. (2.16), the potential energy of the system is given by

$$
\begin{aligned}
\langle V\rangle= & \int d \overrightarrow{\mathbf{r}} \rho(\overrightarrow{\mathbf{r}})\left\{\frac{3}{8} t_{0} \rho(\overrightarrow{\mathbf{r}})+\frac{1}{16} t_{3} \rho^{2}(\overrightarrow{\mathbf{r}})\right\} \\
& +\frac{1}{2} V_{0} \iint \rho(\overrightarrow{\mathbf{r}}) \rho\left(\overrightarrow{\mathbf{r}}^{\prime}\right) \frac{e^{-\left|\overrightarrow{\mathbf{r}}-\overrightarrow{\mathbf{r}}^{\prime}\right| / a}}{\left|\overrightarrow{\mathbf{r}}-\overrightarrow{\mathbf{r}}^{\prime}\right| / a} d \overrightarrow{\mathbf{r}} d \overrightarrow{\mathbf{r}}^{\prime},
\end{aligned}
$$

while the kinetic energy is

$$
\langle T\rangle=4 \sum_{m=1}^{\frac{1}{4} A} \int \psi_{m}^{*}(\overrightarrow{\mathbf{r}})\left(-\frac{\hbar^{2}}{2 m} \nabla^{2}\right) \psi_{m}(\overrightarrow{\mathbf{r}}) d^{3} \overrightarrow{\mathbf{r}} .
$$

The four parameters $t_{0}, t_{3}, V_{0}$, and $a$ appearing in Eqs. (2.16) and (2.18) are adjusted to reproduce the bulk properties of nuclear matter and the surface energy of finite systems. A detailed discussion of their determination is given in the following section.

\section{TWO - BODY EFFECTIVE INTERACTION}

In a discussion of the two-body effective interaction, it is important, at least initially, to distinguish between conceptual and practical difficulties. Conceptually, the static Hartree-Fock problem may be understood in two essentially equivalent ways. The effective interaction may be viewed 
TABLE I. Parameters, nuclear matter properties, and $\mathrm{HF}$ results in ${ }^{16} \mathrm{O}$ and ${ }^{40} \mathrm{Ca}$ for the Skyrme VI force, the Skyrme force with $m^{*}=m$ described in the text, and the finite range Yukawa interaction defined by Eq. (3.2).

\begin{tabular}{lcccc}
\hline \hline & SKM VI & SKM $\left(m^{*}=m\right)$ & Yukawa & (Expt) \\
\hline$t_{0}\left(\mathrm{MeV} \mathrm{fm}^{3}\right)$ & -1101.81 & -1089.0 & -497.726 & \\
$t_{1}\left(\mathrm{MeV} \mathrm{fm}^{5}\right)$ & 271.67 & 251.11 & 0 & \\
$t_{2}\left(\mathrm{MeV} \mathrm{fm}^{5}\right)$ & -138.33 & -150.66 & 0 & \\
$t_{3}\left(\mathrm{MeV} \mathrm{fm}^{6}\right)$ & 17000 & 17270 & 17270 & \\
$a V_{0}(\mathrm{MeV} \mathrm{fm})$ & $\ldots$ & $\ldots$ & -166.9239 & \\
$a(\mathrm{fm})$ & $\ldots$ & $\ldots$ & 0.45979 & \\
$\epsilon_{0}(\mathrm{MeV})$ & -15.77 & -15.77 & -15.77 & \\
$k_{F}\left(\mathrm{fm}^{-1}\right)$ & 1.29 & 1.29 & 1.29 & \\
$K(\mathrm{MeV})$ & 362 & 368 & 368 & \\
$E / A\left[{ }^{16} \mathrm{O}\right](\mathrm{MeV})$ & 7.67 & 7.60 & 8.22 & $(7.98)$ \\
$E / A\left[{ }^{40} \mathrm{Ca}\right](\mathrm{MeV})$ & 8.32 & 8.29 & 8.67 & $(8.55)$ \\
$r_{\mathrm{ch}}\left[{ }^{16} \mathrm{O}\right](\mathrm{fm})$ & 2.72 & 2.72 & 2.67 & $(2.73)$ \\
$r_{\mathrm{ch}}\left[{ }^{40} \mathrm{Ca}\right](\mathrm{fm})$ & 3.48 & 3.48 & 3.45 & $(3.48)$ \\
\hline \hline
\end{tabular}

as a $G$-matrix summation to include the effects of short range correlations. ${ }^{2}$ Alternatively, the entire problem may be viewed as a variation, subject to subsidiary conditions, of a truncated expression for the total energy obtained with a Jastrow wave function. ${ }^{10}$ Both approaches give rise to the same HF theory in which a $G$ matrix, which depends upon the nuclear density matrix, is calculated directly from a realistic two-body force. The nonlocal exchange terms play a significant role in these theories. With modest adjustments to account for higher order processes, such theories yield systematic agreement with the bulk properties of finite nuclei.

For the time-dependent problem, we are not aware of any corresponding treatment of the ef fective interaction. Undoubtedly, the diagrammatic expansion could be formulated in the timedependent basis, with a suitable change in propagators. At reasonably low velocities, the relevant effective interaction should be very similar to the static $G$ matrix. Similarly, since TDHF is usually formulated variationally, a time-dependent generalization ${ }^{11}$ of the Jastrow approach might also be feasible and is likely to yield an effective interaction of the same structure.

Even if the conceptual problems were solved, yielding something like a reaction matrix calculated from a realistic force, strong pragmatic considerations suggest a drastic simplification of the interaction for initial explorations. A wide variety of results indicate that binding energies and radii of finite nuclei may be reproduced with phenomenological interactions of the Skyrme form, which for spin and isospin saturated systems may be written as ${ }^{12}$

$$
\begin{aligned}
v_{\mathrm{SKM}}\left(\overrightarrow{\mathbf{r}}, \overrightarrow{\mathbf{r}}^{\prime}\right)= & t_{0} \delta\left(\overrightarrow{\mathbf{r}}-\overrightarrow{\mathbf{r}}^{\prime}\right) \\
& +\frac{1}{2} t_{1}\left[\delta\left(\overrightarrow{\mathbf{r}}-\overrightarrow{\mathbf{r}}^{\prime}\right) k^{\prime 2}+k^{2} \delta\left(\overrightarrow{\mathbf{r}}-\overrightarrow{\mathbf{r}}^{\prime}\right)\right] \\
& +t_{2} \overrightarrow{\mathbf{k}} \cdot \delta\left(\overrightarrow{\mathbf{r}}-\overrightarrow{\mathbf{r}}^{\prime}\right) \overrightarrow{\mathrm{k}}^{\prime}+\frac{1}{6} t_{3} \rho \delta\left(\overrightarrow{\mathbf{r}}-\overrightarrow{\mathbf{r}}^{\prime}\right) .
\end{aligned}
$$

Here $\overrightarrow{\mathrm{k}}^{\prime}=\left(\nabla_{r}-\nabla_{r^{\prime}}\right) / \mathbf{2} i$ acts to the right and $\overrightarrow{\mathrm{k}}=\left(\nabla_{r^{\prime}}\right.$ $\left.-\nabla_{r}\right) / 2 i$ acts to the left. Using the fact that one combination of the Skyrme parameters, the range of nonlocality or equivalently the effective mass $m *$, is virtually undetermined by binding energies and radii, one is free to choose a purely local effective interaction which results in significant simplification of the slab problem. The force Skyrme VI is very close to being local, with $m *$ / $m=0.95$ in nuclear matter. ${ }^{5}$ A small readjustment of the parameters yields a Skyrme force with the same volume energy, saturation density, surface energy, and with $m * / m=1.0$. Because the remaining combination of $t_{1}$ and $t_{2}$ simply describes the finite range direct interaction, it is more physical to replace this term by a finite range Yukawa interaction with a realistic range and a strength adjusted to reproduce the same surface energy. Thus, we add the potential

$$
v_{\text {YUK }}(r)=V_{0} \frac{e^{-r / a}}{r / a} P .
$$

Here $V_{0}=\left(3 t_{1}-5 t_{2}\right) / 64 \pi a^{5}$, the operator $P=\frac{16}{15}+\frac{4}{15} P_{x}$, with $P_{x}$ the space exchange operator, restricts $v_{\text {YUK }}$ to contribute only to the direct term, and the range $a=0.45979 \mathrm{fm}$ is obtained by fitting to a realistic direct interaction. ${ }^{13}$ In addition, such a Yukawa offers the technical advantage that the integration of the TDHF equations is much more stable with a finite range force than with a gradient expansion. The parameters, nuclear matter properties, and 

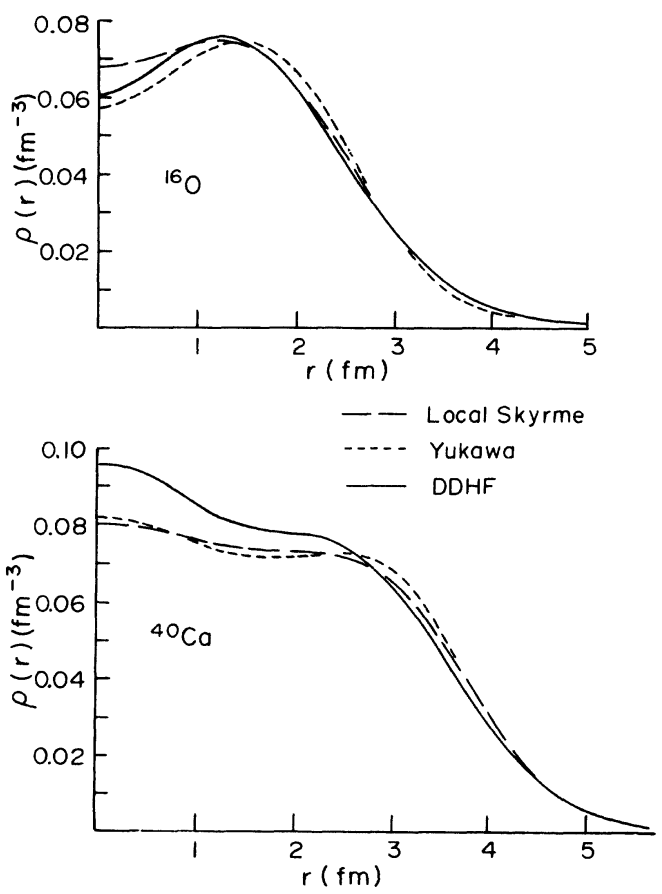

FIG. 1. Proton density distributions for ${ }^{16} \mathrm{O}$ and ${ }^{40} \mathrm{Ca}$ calculated with the local Skyrme $\left(m^{*}=m\right)$ and Yukawa forces listed in Table I. For comparison, the proton density given by the density-dependent Hartree-Fock calculation of Ref. 2 is also shown.

finite nucleus results for SKM VI, the Skyrme force with $m * / m=1$, and our Yukawa force are shown in Table I. Since our interaction is designed for spin and isospin saturated systems, it is only possible to test it in light mirror nuclei. From the binding energies and radii in Table $I$, it is evident that the gross structure of these nuclei is reasonably well reproduced. The actual proton density distributions are somewhat less accurately represented, as shown in Fig. 1, where comparison is made with density-dependent HF distributions which fit elastic electron scattering. ${ }^{2}$ The origin of the discrepancy in ${ }^{40} \mathrm{Ca}$ appears to be the fact that $m * / m=1$ Skyrme and Yukawa parameters were adjusted to reproduce the nuclear matter and surface properties of SKM VI. The force SKM VI itself does not reproduce the ${ }^{40} \mathrm{Ca}$ density very accurately, yielding results visually indistinguishable from the long dashed lines in Fig. 1. Presumably by suitable readjustment of the nuclear matter saturation density, a more reasonable ${ }^{40} \mathrm{Ca}$ density could be obtained, and there is no fundamental deficiency in the form of parametrization selected.

In summary, the interaction we have used is admittedly schematic, and intended for exploratory calculations only. Its most serious deficiency is the assumption of $m * / m=1$, given that we know that a realistic nuclear force yields significant nonlocality in the self-consistent field. ${ }^{14}$ This is particularly relevant to the time-dependent problem, because it is precisely the exchange term which contains the contribution of the current to the HF potential. This connection is especially clear in the case of the Skyrme interaction, where the general Hamiltonian density contains, in addition to the terms we have retained, the term ${ }^{15}$

$$
\frac{1}{10}\left(3 t_{1}+5 t_{2}\right)\left(\rho \tau-J^{2}\right),
$$

where

$$
\tau(\overrightarrow{\mathbf{r}})=\left[\vec{\nabla} \cdot \vec{\nabla}^{\prime} \rho\left(\overrightarrow{\mathbf{r}}, \overrightarrow{\mathbf{r}}^{\prime}\right)\right]_{\vec{r}}=\vec{r}^{\prime},
$$

and

$$
J(\overrightarrow{\mathrm{r}})=\frac{1}{2} i\left[\left(\vec{\nabla}-\vec{\nabla}^{\prime}\right) \rho\left(\overrightarrow{\mathrm{r}}, \overrightarrow{\mathbf{r}}^{\prime}\right)\right]_{\overrightarrow{\mathrm{r}}=\overrightarrow{\mathrm{r}}^{\prime}} .
$$

For example, the exchange term is responsible for the difference between the Thouless-Valatin and cranking formulas for inertial parameters and in the realistic case of $m * / m=0.7$ gives rise to a $30 \%$ difference. ${ }^{16}$ Hence the exchange term may play a significant role in nuclear dynamics and certainly requires attention in subsequent investigations.

\section{SPECIALIZATION TO SLAB GEOMETRY}

In principle, we would like to integrate $\frac{1}{4} A$ coupled three-dimensional equations (2.9) with some set of physically interesting initial conditions. However, even three-dimensional hydrodynamic calculations which involve integrating four coupled equations in a three-dimensional space strain the present numerical capabilities. ${ }^{17}$ Thus we must limit the dimensionality of our calculation. This section discusses the specialization of the three-dimensional TDHF equations (2.9) to a situation of slab geometry. We first treat the static HF problem, discussing the gross features and static HF solutions of slabs. We then present the specialized TDHF equations to be solved in this geometry.

\section{Gross features of static slabs}

The type of systems we wish to consider are uncharged slabs of nuclear matter. These slabs are infinite and homogeneous in the two transverse directions and finite in the $z$ coordinate.

When the slab thickness is sufficiently large that the surfaces are well separated, several features are evident.

(i) Because of nuclear matter saturation, the ground state density profile $\rho(z)$ consists of a large central region of almost constant density $\rho_{0}$. On either side of this central region, there is a 
surface region in which the density falls from $\rho_{0}$ to zero over a distance of $\sim 2 \mathrm{fm}$. The shape of this transition region should be independent of the thickness of the slab. For thin slabs, these features are modified because of the proximity of the two surface regions and this is the reason why our present discussion is limited to thick slabs.

(ii) Slabs have varying thickness $L$ defined as the distance between the half density points $\left(\frac{1}{2} \rho_{0}\right)$ in either surface. It will be useful to measure this thickness by the number of nucleons per unit area in the $x-y$ plane, defined as

$$
\Theta=\int_{-\infty}^{\infty} \rho(z) d z .
$$

Because of saturation, we have

$$
Q \sim \rho_{0} L .
$$

(iii) The slab binding energy may also be determined from macroscopic properties. Because the slab is infinite in the transverse directions, the relevant quantity is the energy per unit area. This energy is given by a volume and a surface term

$$
E / \Omega=E_{\mathrm{vol}} / \Omega+E_{\text {surf }} / \Omega,
$$

where $\Omega$ is an infinite normalizing area in the $x-y$ plane. Since the energy per nucleon in nuclear matter is $\epsilon_{0}$, the (negative) volume energy per unit area is

$$
E_{\mathrm{vol}} / \Omega=Q \epsilon_{0} .
$$

In finite nuclei, the (positive) surface energy per unit area from the semiempirical mass formula is $a_{s} / 4 \pi r_{0}^{2}$, where $a_{s}$ is the surface energy coefficient, ${ }^{18}$ and

$$
\frac{4 \pi}{3} r_{0}^{3}=\frac{1}{\rho_{0}} \text {. }
$$

Thus, for a slab

$$
\frac{E_{\text {surf }}}{\Omega}=\frac{a_{s}}{2 \pi r_{0}^{2}},
$$

where the extra factor of 2 accounts for the two surfaces of the slab. Therefore,

$$
\frac{E}{\Omega}=\epsilon_{0} \mathfrak{Q}+\frac{a_{s}}{2 \pi r_{0}^{2}} \text {. }
$$

In the limit of large $a$, the binding energy per particle and average density of the slab referred to above are determined directly from nuclear matter quantities. Using the Yukawa interaction introduced in Sec. III, the nuclear matter binding energy per nucleon is

$$
\frac{E}{A}=\frac{3}{10}\left(\frac{3 \pi^{2}}{2}\right)^{2 / 3} \frac{\hbar^{2}}{m} \rho^{2 / 3}+\left(\frac{3}{8} t_{0}+2 \pi a^{3} V_{0}\right) \rho+\frac{1}{16} t_{3} \rho^{2},
$$

the saturation density $\rho_{0}$ is given by the relation

$$
\frac{\partial}{\partial \rho}(E / A)_{\rho=\rho_{0}}=0,
$$

and the compression modulus is

$$
\begin{aligned}
K & =\left.9 \rho_{0}{ }^{2} \frac{\partial^{2}(E / A)}{\partial \rho^{2}}\right|_{\rho=\rho_{0}} \\
& =\frac{6}{5}\left(\frac{3 \pi^{2}}{2}\right)^{2 / 3} \frac{\hbar^{2}}{m} \rho_{0}{ }^{2 / 3}-18 \epsilon_{0} .
\end{aligned}
$$

This latter quantity is crucial, since only compressional modes are possible in our slab geometry.

(iv) In analogy with real nuclei, one might expect slabs to exhibit shell effects in both binding energies and density profiles. Quantum density fluctuations in $\rho(z)$ will occur because of the quantization associated with the finite extent of the slab in the $z$ direction. Shell fluctuations in the binding energies of real nuclei, however, are associated with the existence of gaps in the singleparticle energy spectrum. For the case of slabs, as elaborated below, translation invariance in the transverse directions gives rise to a continuous spectrum, so that no gaps and thus no energy fluctuations arise.

\section{Static Hartree-Fock}

In this section we discuss the static HF equations, the solutions of which are used to construct the initial conditions for physically interesting problems.

Since the density is a function of $z$ only, the $\mathrm{HF}$ potential $W$ defined by Eq. (2.16) will also depend only upon $z$. After integration over transverse coordinates, we obtain

$$
\begin{aligned}
W(z)= & \frac{3}{4} t_{0} \rho(z)+\frac{3}{16} t_{3} \rho^{2}(z) \\
& +2 \pi a^{2} V_{0} \int_{-\infty}^{\infty} d z^{\prime} \rho\left(z^{\prime}\right) e^{-\left|z-z^{\prime}\right| / a} .
\end{aligned}
$$

With this potential, the time-independent Schrödinger equation

$$
\left[-\frac{\hbar^{2}}{2 m} \nabla_{\overrightarrow{\mathrm{r}}}^{2}+W(z)\right] \psi_{i}(\overrightarrow{\mathrm{r}})=\epsilon_{i} \psi_{i}(\overrightarrow{\mathrm{r}})
$$

is separable. Each spatial wave function $\psi_{i}(\overrightarrow{\mathrm{r}})$ is then a product of a plane wave in the transverse coordinates $\left(\overrightarrow{\mathbf{r}}_{\perp}\right)$ and a wave function in the $z$ coordinate

$$
\psi_{i}(\overrightarrow{\mathrm{r}})=\psi_{n \overrightarrow{\mathrm{k}}_{\perp}}(\overrightarrow{\mathrm{r}})=\frac{1}{\sqrt{\Omega}} e^{i \overrightarrow{\mathrm{k}}_{\perp} \cdot \overrightarrow{\mathrm{r}}} \perp \phi_{n}^{\mathrm{HF}}(z) .
$$

The $\phi$ 's are normalized to

$$
\int_{-\infty}^{\infty}\left|\phi_{n}^{\mathrm{HF}}(z)\right|^{2} d z=1 .
$$


The subscript $i$ labels both the $z$ quantum number $n$ and the transverse wave vector $\overrightarrow{\mathrm{k}}_{\perp}$. From Eq. (4.12), the single-particle energy is given by

$$
\epsilon_{i}=\epsilon_{n k_{\perp}}=e_{n}+\frac{\hbar^{2}}{2 m} k_{\perp}^{2}
$$

where $e_{n}$ is determined by the bound state Schrödinger equation

$$
\left[-\frac{\hbar^{2}}{2 m} \frac{d^{2}}{d z^{2}}+W(z)\right] \phi_{n}^{\mathrm{HF}}(z)=e_{n} \phi_{n}^{\mathrm{HF}}(z) .
$$

In order to construct a $\mathrm{HF}$ solution for a slab, we have to self-consistently solve Eq. (4.12). The ground state will correspond to the occupation of all states $\psi_{n k_{\perp}}$ with energy $\epsilon_{n k_{\perp}}$ below the Fermi energy $\epsilon_{F}$ :

$$
\epsilon_{n k_{\perp}}=e_{n}+\frac{\hbar^{2}}{2 m} k_{\perp}^{2} \leqslant \epsilon_{F}
$$

or

$$
\left|\overrightarrow{\mathrm{k}}_{\perp}\right|<\left[\left(2 m / \hbar^{2}\right)\left(\epsilon_{F}-e_{n}\right)\right]^{1 / 2} \equiv k_{\max }(n) .
$$

Therefore, each $\phi_{n}$ is associated with plane waves of transverse momentum within a circle in the $\overrightarrow{\mathrm{k}}_{\perp}$ plane of radius $k_{\max }(\boldsymbol{n})$. The Fermi energy (or chemical potential) is given implicitly by the size of the slab we want to construct.

With the orbitals occupied as described above, the density is given by

$$
\begin{aligned}
\rho(z) & =4 \sum_{\substack{n, k_{\perp} \\
\text { occupied }}}\left|\psi_{n k_{\perp}}(\overrightarrow{\mathbf{r}})\right|^{2} \\
& =4 \sum_{\substack{n \\
\text { occupied }}}\left|\phi_{n}^{\mathrm{HF}}(z)\right|^{2} \int_{0}^{k_{\max }(n)} \frac{d^{2} k_{\perp}}{(2 \pi)^{2}} \\
& =\sum_{\substack{n \\
\text { occupied }}}\left|\phi_{n}^{\mathrm{HF}}(z)\right|^{2} \frac{k_{\max }{ }^{2}(n)}{\pi} .
\end{aligned}
$$

We may define

$$
Q_{n}=\frac{k_{\max }^{2}(n)}{\pi}=\frac{2 m}{\hbar^{2}} \frac{\epsilon_{F}-e_{n}}{\pi},
$$

so that

$$
\rho(z)=\sum_{\substack{n \\ \text { occupied }}} \mathcal{Q}_{n}\left|\phi_{n}^{\mathrm{HF}}(z)\right|^{2} .
$$

Thus, each orbital $\phi_{n}^{\mathrm{HF}}$ carries an effective normalization proportional to the difference between its eigenvalue $e_{n}$ and the Fermi energy $\epsilon_{F}$. For a nonlocal HF potential (e.g., $m * / m \neq 1$ ), the transverse plane waves are coupled to the longitudinal wave functions, and one would have to solve for $\phi_{n}\left(\overrightarrow{\mathrm{k}}_{\perp}, z\right)$. It is because of this substantial complication that we have restricted our attention to purely local HF potentials (i.e., local exchange potential or $m *=m$ ) for which the transverse plane waves affect the problem only through weighting factors, as in Eq. (4.21). Thus, while the structure of the resulting problem is no more difficult computationally than a genuine one-dimensional problem, we may utilize a three-dimensional interaction and three-dimensional phase space.

According to Eqs. (4.20) and (4.21), $\mathcal{G}$ is given by

$$
\mathcal{Q}=\sum_{n=1}^{N} \mathfrak{Q}_{n}=\sum_{n=1}^{N} \frac{2 m}{\pi \hbar^{2}}\left(\epsilon_{F}-e_{n}\right),
$$

where $N$ is the number of occupied bound orbitals $\phi_{n}(z)$. We must have

$$
e_{N+1}>\epsilon_{F},
$$

so that no gaps occur in the spectrum of occupied single-particle levels.

For a given value of $\mathcal{Q}$, Eqs. (4.22) and (4.23) determine $\epsilon_{F}$, given a set of eigenvalues $e_{n}$. Thus Eqs. (4.11), (4.16), (4.20), (4.21), (4.22), and (4.23) provide the set of HF equations to be solved self-consistently for a given $Q$.

Once a consistent solution has been obtained, the energy per unit area is given by

$$
\begin{aligned}
\frac{E}{\Omega}= & \frac{\hbar^{2}}{2 m} \sum_{n=1}^{N} G_{n} \int_{-\infty}^{\infty}\left|\frac{d \phi_{n}^{\mathrm{HF}}(z)}{d z}\right|^{2} d z+\sum_{n=1}^{N} \frac{\hbar^{2}}{4 m} \pi{G_{n}{ }^{2}}^{\infty} \\
& +\frac{3}{8} \int_{-\infty}^{\infty} t_{0} \rho^{2}(z) d z+\frac{t_{3}}{16} \int_{-\infty}^{\infty} \rho^{3}(z) d z \\
& +\pi a^{2} V_{0} \int_{-\infty}^{\infty} d z \int_{-\infty}^{\infty} d z^{\prime} \rho(z) \rho\left(z^{\prime}\right) e^{-\left|z-z^{\prime}\right| / a} .
\end{aligned}
$$

The description of a slab of substantial thickness requires a surprisingly small value of $N$. The last occupied orbital $\phi_{N}^{\mathrm{HF}}$ is bound in a slab with an internal momentum of $\sim k_{F}$, the Fermi momentum in nuclear matter. For a slab of thickness $L$ the number of nodes in $\phi_{N}^{\mathrm{HF}}$ will be

$$
\frac{k_{F} L}{\pi}-1
$$

Therefore, we may estimate $N$ by

$$
N \sim \frac{k_{F} L}{\pi}
$$

For a slab of $L=10 \mathrm{fm}$ thickness which has $Q \sim 1.5$ $\mathrm{fm}^{-2}, N$ is equal to 4 . The four wave functions represent the only accessible degrees of freedom, since the transverse coordinates are decoupled and thus effectively frozen.

The numerical solution of the static HF equa- 


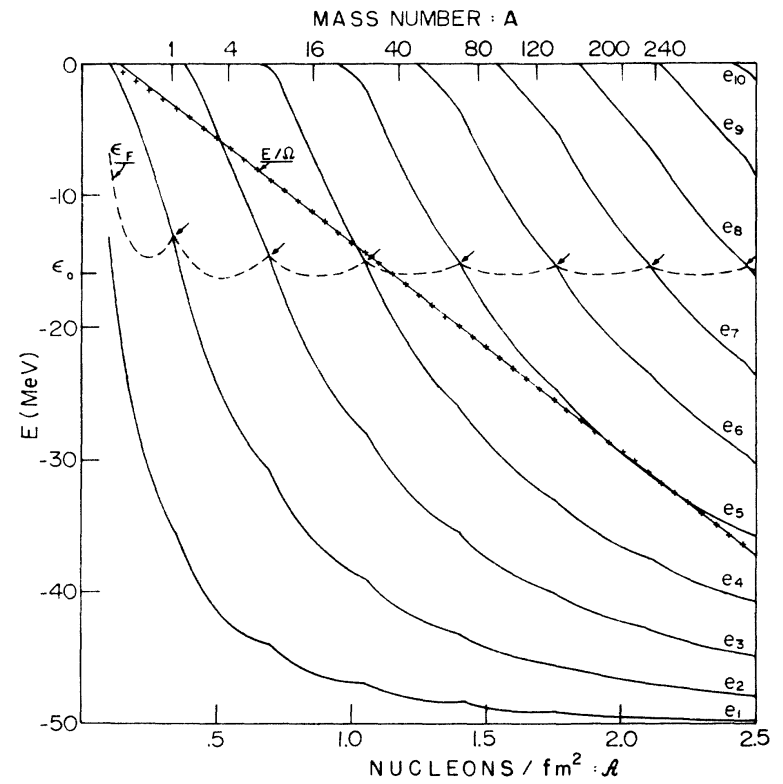

FIG. 2. The slab mass table. The solid lines show the eigenvalues $e_{n}$ of Eq. (4.16) as a function of slab thickness $a$. The dashed curve indicates the fermi energy $\epsilon_{F}$, with arrows giving the "magic" slabs. The crosses show the calculated slab energy $E / \Omega$ in units of $\mathrm{MeV} \mathrm{fm}{ }^{-2}$, which has been fitted to a straight line over the region $Q \geq 0.1 \mathrm{fm}^{-2}$ as per Eq. (4.7). The upper abscissa is labeled by the three-dimensional equivalent mass number given by Eq. (4.27). tions has been performed by iteration. The details are described in Appendix A.

\section{Static results}

Figure 2 summarizes the slab mass table which is obtained with the force parameters listed in Table I. We plot various properties of the static solutions as a function of $G$. The solid lines labeled by $e_{n}$ denote the eigenvalues of Eq. (4.16). The dotted line is the Fermi energy $\epsilon_{F}$. "Magic" slabs occur when a new eigenvalue dives below $\epsilon_{F}$. These slabs are characterized by cusps both in $\epsilon_{F}$ and in the $e_{n}$ 's at the values of $Q$ indicated by arrows. The "shell effects" are, of course, more prominent for small $Q$. As expected from the Hugenholtz-Van Hove theorem, the Fermi energy oscillates about $\epsilon_{0}$ for large $Q$.

Figure 2 also summarizes the binding energy per $\mathrm{fm}^{2}(E / \Omega)$ as a function of $\mathrm{A}$ (crosses). It is smooth and well represented by a linear function for $Q>0.3 \mathrm{fm}^{-2}$. The solid line corresponds to a least squares fit over the region $Q>0.1 \mathrm{fm}^{-2}$ and results in the values of volume and surface energies listed in Table I. The deviation from this straight line for small $G$ is due to the overlap of the two surface regions, which invalidates Eq. (4.7).

While the transcription of the one-dimensional systems we are treating to realistic three-dimen-

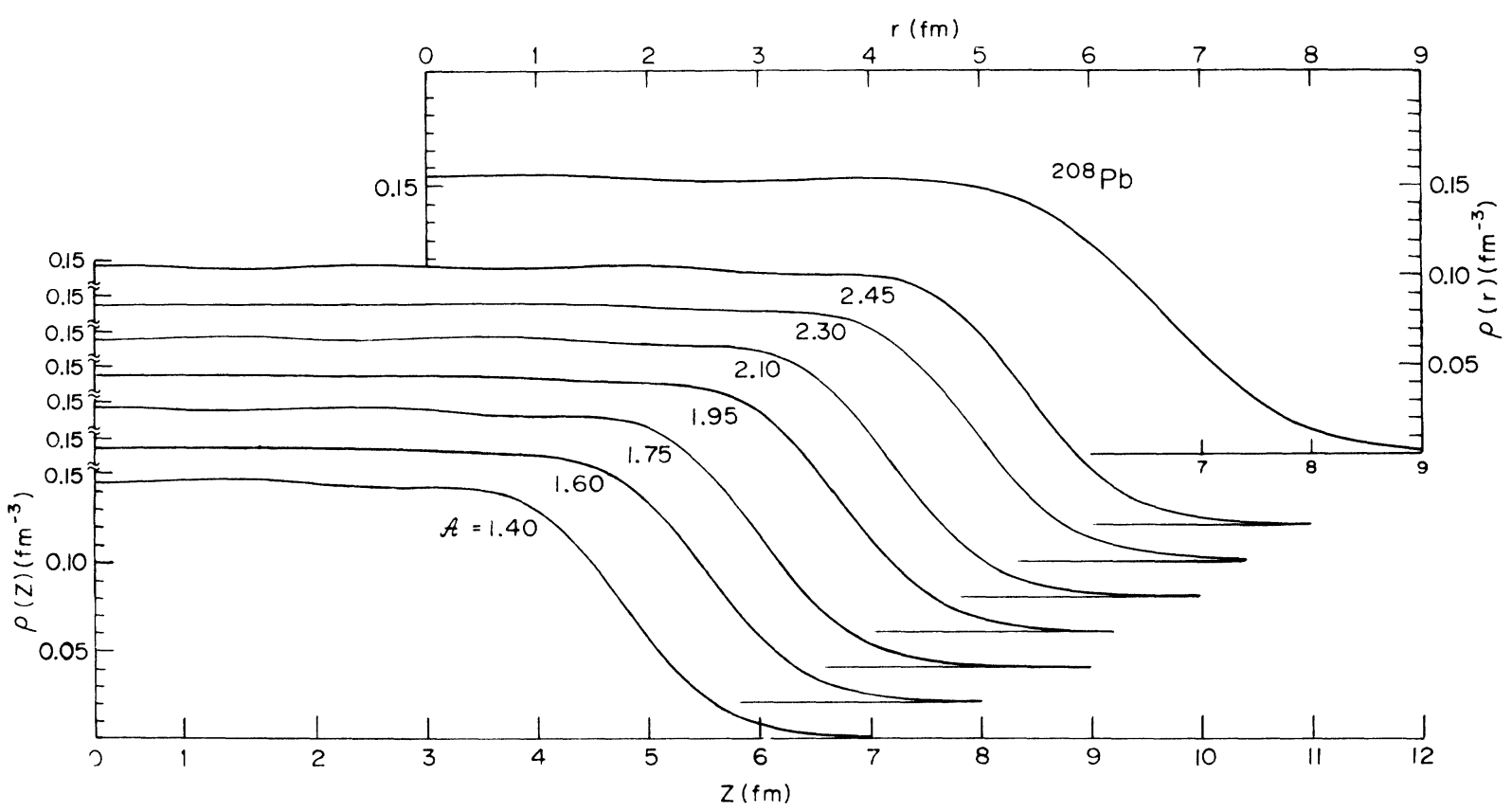

FIG. 3. Density profiles for static slabs of varying thickness. Shown for comparison is the density profile of ${ }^{208} \mathrm{~Pb}$ calculated in Ref. 2 . 
sional nuclei is ambiguous, the scale is set by the diameter of a nucleus of mass $A$ :

$$
Q \sim 2 r_{0} A^{1 / 3} \rho_{0},
$$

A sequence of static slab profiles, $\rho(z)$, is presented in Fig. 3 for increasing values of $G$. Because of reflection symmetry with respect to the slab centers, only half of each distribution is plotted. It is observed that the interior density is systematically very close to the nuclear matter value, $\rho_{0}=0.145 \mathrm{fm}^{-3}$ and that the thickness increases linearly with $Q$ as expected. The quantum density fluctuations, ${ }^{19}$ however, are quite different in structure from those observed in spherical finite nuclei, and this may be understood from Eq. (4.21) for $\rho(z)$. The weighting factors $Q_{n}$ decrease as $e_{n}$ approaches $\epsilon_{F}$, so that the discontinuity in wave function weighting at the Fermi surface is much weaker for slabs than for spherical nuclei. It is this discontinuity which gives rise to density oscillations. The greatest discontinuity occurs when the last nonzero $Q_{n}$ has its maximum value, which occurs when $e_{N+1}$ is just slightly greater than $\epsilon_{F}$. In these cases the density fluctuations should be strongest. However, if $\epsilon_{F}$ is midway between $e_{N}$ and $e_{N+1}$, the discontinuity in wave function weighting is much weaker and the density fluctuations are expected to be correspondingly diminished. These expectations are borne out in Fig. 3. The fluctuations are significant for the cases $Q=1.40,1.75,2.10$, and $2.45 \mathrm{fm}^{-2}$, which from Fig. 2 correspond to the intersection of eigenvalues $n=5$ through 8 with $\epsilon_{F}$. However, for the cases $Q=1.60,1.95$, and $2.30 \mathrm{fm}^{-2}$, which were selected such that $\epsilon_{F}$ was roughly midway between eigenvalues, no fluctuations occur in the central region.

One may also observe that the wavelength of density fluctuation agrees with the expected value of $\pi / k_{F}=1.43 \mathrm{fm}$ and that the presence of a central maximum or minimum is correctly specified by the parity of the last occupied orbital. In the limit of large $a$, the magnitude of the interior oscillations should diminish as $1 / Q$. Since the level density for $e_{n}$ approaches a continuum, in this limit only the fluctuations near the surface remain. These arise from the fact that $Q(e) \equiv \mathbf{2} m\left(\epsilon_{F}-e\right) /$ $\pi \hbar^{2}$ approaches zero linearly at $\epsilon_{F}$.

For comparison with real nuclei, the matter distribution of ${ }^{208} \mathrm{~Pb}$ is also included in Fig. 3. The distribution is taken from the calculation of Ref. 2 for which the proton distribution agrees well with elastic electron scattering data. The interior density and surface thickness are quite comparable with those obtained for slabs. We do not wish to push the comparison too far, since the Coulomb interaction, neutron excess, and surface tension play significant roles in the structure of ${ }^{208} \mathrm{~Pb}$. In view of the previous discussion of density fluctuations, it should be noted that the reason for the anomalously smooth density in ${ }^{208} \mathrm{~Pb}$ is the fact that the substantial neutron and proton density fluctuations happen to be completely out of phase in this nucleus.

\section{Dynamic equations}

We now discuss the specialization of the general equations $[(2.5)-(2.9)]$ governing the time evolution of the single-particle wave functions to the case of slab symmetry with our particular effective interaction. As expected, the static HF equations $[(4.11),(4.16),(4.21)]$ find simple dynamical generalizations.

Since the density has been constrained to depend only upon $z$ at all time, the HF potential $W$ given by Eq. (4.11) also depends only upon this variable. Therefore, the transverse plane wave component of each single-particle wave function remains constant in time. Indeed, from the Hamiltonian in Eq. (4.12), the replacement of $e_{i}$ by $i \hbar \partial / \partial t$ shows that the plane wave only contributes a physically unimportant over-all phase to $\psi_{n k_{\perp}}$, given by $\exp \left(-i \hbar k_{\perp}{ }^{2} t / 2 m\right)$. All the important time-dependence then resides in the $\phi_{n}(z, t)$. Defining

$$
\psi_{n k_{\perp}}(\overrightarrow{\mathrm{r}}, t)=e^{-i \hbar_{k_{\perp}}{ }^{2} t / 2 m} \frac{e^{i \overrightarrow{\mathrm{k}}_{\perp} \cdot \overrightarrow{\mathrm{r}}_{\perp}}}{\sqrt{\Omega}} \phi_{n}(z, t),
$$

we obtain

$i \hbar \dot{\phi}_{n}(z, t)=\left(-\frac{\hbar^{2}}{2 m} \frac{\partial^{2}}{\partial z^{2}}+W(z, t)\right) \phi_{n}(z, t)$,

i.e., a one-dimensional Schrödinger equation with time-dependent potential $W(z, t)$. Note that because the transverse plane waves are invariant in time the transverse occupations of the $\phi_{n}$, specified by the weights $Q_{n}$, are constant in time. As seen in the following section, the initial conditions for our TDHF solutions are obtained from static HF solutions, so that the $a_{n}$ 's are specified by the initial conditions. The many-body wave function thus has no freedom to adjust transverse occupation, so that the transverse coordinates are decoupled. This rather severe limitation, which must be imposed for reasons of numerical tractability, has strong implications regarding the physical applicability of our calculations. These are discussed in detail in Sec. VIII.

In several of the calculations discussed below, we have investigated the effect of a static, spinisospin-independent external potential. This has been accomplished by the addition of the potential $V_{\text {ext }}(z)$ to $W(z)$ in Eq. (4.29), where the functional form of $V_{\text {ext }}$ is discussed in Sec. V. This potential entails the addition of a term 


$$
\frac{\left\langle V_{\text {ext }}\right\rangle}{\Omega}=\int_{-\infty}^{\infty} V_{\mathrm{ext}}(z) \rho(z) d z
$$

to Eq. (4.24) for the total energy $E / \Omega$.

\section{INITIAL CONDITIONS AND SINGLE SLAB DYNAMICS}

This section and the following present solutions of the TDHF equations in slab geometry for a variety of increasingly complex situations. We begin here with a discussion of initial conditions. The small amplitude oscillations of a single slab induced by an initially coherent velocity field are then discussed. Finally, we treat the interaction of a single slab with an external potential.

\section{Initial conditions}

Physically plausible initial conditions may be generated for all situations by the application of a coherent velocity field (the same for all wave functions) to the static HF solutions. From the Gallilean invariance of the TDHF equations, it follows that if the static HF wave functions for a slab of thickness $Q$ are all multiplied by the common phase factor

$$
\phi_{n}(z, t=0)=\phi_{n}^{\mathrm{HF}}(z) e^{i k z},
$$

where $k$ is an arbitrary wave number, the resultant evolution of the system will be uniform translation with the velocity

$$
\begin{aligned}
v(z, t) & =\frac{1}{\rho(z, t)} \frac{\hbar}{m} \sum_{n} \boldsymbol{Q}_{n} \operatorname{Im}\left(\phi_{n}^{*} \frac{\partial \phi_{n}}{\partial z}\right) \\
& =\hbar k / m .
\end{aligned}
$$

Note that Eq. (5.2a) defines the velocity field as that which is associated with the quantum mechanical probability current. By uniform translation we mean, of course, translation of observables such as the density and not translation of the wave functions themselves, which will acquire inconsequential time-dependent phases. From Eq. (4.24) the conserved energy of the system is then

$$
\frac{E}{\Omega}=\frac{E^{\mathrm{HF}}}{\Omega}+Q \frac{\hbar^{2} k^{2}}{2 m},
$$

where $E^{\mathrm{HF} / \Omega}$ is the static HF binding energy per unit area. Thus, the initial conditions (5.1) result in a solution corresponding to the uniform translation of a slab in its ground state.

The extension of Eq. (5.1) to the case of colliding slabs is now straightforward. For a collision between two slabs of mass $Q_{1}$ and $Q_{2}$ with bombarding energy $E / A$ per nucleon in the center of mass system, the static HF solutions are placed on a mesh, far enough apart so that their overlap is negligible (the inner products of $\phi$ 's associated with different slabs being $\left.\leqslant 10^{-6}\right)$. The static wave functions comprising the $Q_{1}$ solution are then multiplied by the phase $e^{i k_{1} z}$ with

$$
k_{1}=\left[\frac{2 m}{\hbar^{2}}\left(\frac{E}{A}\right) \frac{Q_{2}}{Q_{1}}\right]^{1 / 2},
$$

while those of the $Q_{2}$ solution are multiplied by the phase $e^{i k_{2} z}$ :

$$
k_{2}=-\frac{\boldsymbol{Q}_{1}}{\boldsymbol{Q}_{2}} k_{1}=-\left[\frac{2 m}{\hbar^{2}}\left(\frac{E}{A}\right) \frac{\boldsymbol{Q}_{1}}{\boldsymbol{Q}_{2}}\right]^{1 / 2} .
$$

These initial conditions correspond to the required situation, with the collision taking place in the c.m. system frame.

The energy $E / A$ is the center of mass bombarding energy per particle, i.e., the total bombarding energy divided by the total number of particles. For a collision $Q_{1}+G_{2}$, the bombarding energy per particle of the projectile in the laboratory system is given by

$$
\left(\frac{E}{A}\right)_{\mathrm{lab}}=\frac{\left(\boldsymbol{Q}_{1}+Q_{2}\right)^{2}}{\mathcal{Q}_{1} \boldsymbol{Q}_{2}}\left(\frac{E}{A}\right)_{\text {c.m. }} .
$$

For symmetric collisions where $\boldsymbol{Q}_{1}=\boldsymbol{Q}_{2}$, we have

$$
\left(\frac{E}{A}\right)_{\mathrm{lab}}=4\left(\frac{E}{A}\right)_{\text {c.m. }} .
$$

Hereafter we will always refer to the c.m. value of $E / A$.

The generalization of Eq. (5.1) to arbitrary velocity fields is straightforward. ${ }^{20}$ Indeed, the initial conditions

$$
\phi_{n}(z, t=0)=\phi_{n}^{\mathrm{HF}}(z) e^{i S(z)},
$$

where $S(z)$ is any real function of $z$, result in a coherent velocity field at $t=0$ given by Eq. (5.2a) as

$$
v(z)=\frac{\hbar}{m} \frac{d S}{d z} .
$$

Small amplitude dynamics

The small amplitude dynamics of slabs about the static HF solutions provides a useful introduction to the semiclassical behavior characteristic of the TDHF equations, as well as illuminating concepts useful in the analysis of more complicated situations. In order to investigate the oscillations of a single slab, we have used the initial conditions (5.8) with $S(z)=\alpha z^{2}, \alpha$ being a constant. This corresponds to the excitation of a mean-square length breathing mode, the one-dimensional ana$\log$ of monopole vibrations. The initial velocity field is then given by Eq. (5.9) as

$$
v(z)=2 \alpha \hbar z / m \text {. }
$$

A convenient measure of the strength of excitation is the excitation energy per nucleon 


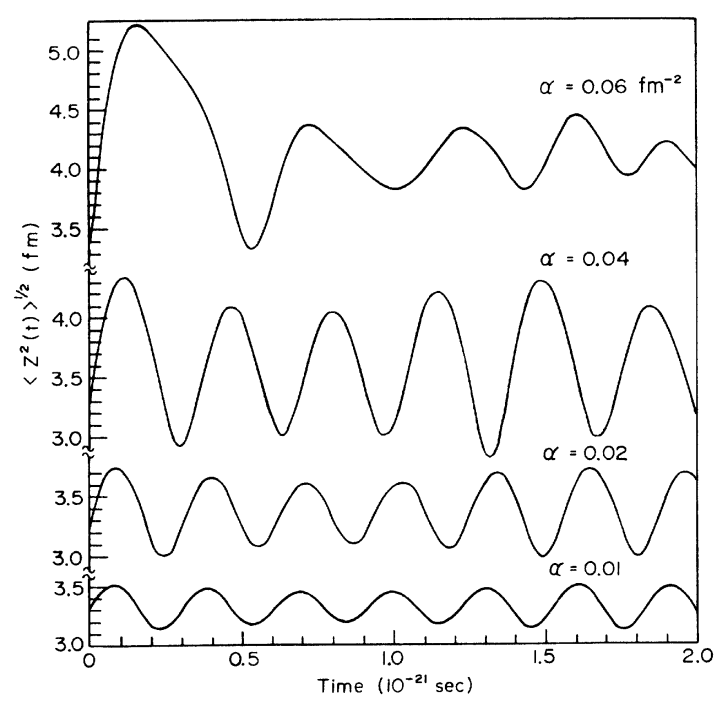

FIG. 4. Root-mean-square lengths of $Q=1.6 \mathrm{fm}^{-2}$ slabs as a function of time. The initial conditions are specified by Eq. (5.10) with the indicated values of $\alpha$.

$$
\frac{E^{*}}{A}=\frac{m}{2 Q} \int_{-\infty}^{\infty} v^{2}(z) \rho(z) d z=2 \frac{\hbar^{2}}{m} \alpha^{2}\left\langle z^{2}\right\rangle,
$$

where the mean-square length of the slab is

$$
\begin{aligned}
& \left\langle z^{2}\right\rangle=\frac{1}{Q} \int_{-\infty}^{\infty} z^{2} \rho(z) d z \\
& \sim L^{2} / 12=Q^{2} / 12 \rho_{0}^{2} .
\end{aligned}
$$

The gross features of the dynamics of the cases we have investigated are succinctly displayed by the time dependence of the mean-square length defined by Eq. (5.12a). Figure 4 shows $\left\langle z^{2}(t)\right\rangle^{1 / 2}$ for excitations of a slab of thickness $Q=1.6 \mathrm{fm}^{-2}$ $(N=5$ from Fig. 2), with strengths $\alpha=0.01,0.02$, 0.04 , and $0.06 \mathrm{fm}^{-2}$ corresponding to the energies $E * / A$ tabulated in Table II.

The oscillations in $\left\langle z^{2}\right\rangle^{1 / 2}$ correspond to the classically expected "breathing mode" oscillations of the slab. In the three cases of weakest excitation $\left(\alpha=0.01,0.02\right.$, and $\left.0.04 \mathrm{fm}^{-2}\right)$, the meansquare length oscillates about the ground state value. However, these oscillations do not appear to be associated with a single frequency but instead

TABLE II. Frequencies $\hbar \omega_{1}$ and $\hbar \omega_{2}$ of the oscillation modes observed in Fig. 4 and the excitation energies $E^{*} / A$ defined in Eq. (5.11) for the $Q=1.6 \mathrm{fm}^{-2}$ system.

\begin{tabular}{cccc}
\hline \hline$\alpha\left(\mathrm{fm}^{-2}\right)$ & $E^{*} / A(\mathrm{MeV})$ & $\hbar \omega_{1}(\mathrm{MeV})$ & $\hbar \omega_{2}(\mathrm{MeV})$ \\
\hline 0.01 & 0.08 & $11.08 \pm 0.07$ & $15.95 \pm 0.07$ \\
0.02 & 0.34 & $10.54 \pm 0.09$ & $16.06 \pm 0.09$ \\
0.04 & 1.35 & $9.1 \pm 0.2$ & $15.0 \pm 0.2$ \\
0.06 & 3.03 & & \\
\hline \hline
\end{tabular}

may be interpreted in terms of a beating of two modes. The energies associated with these modes $(\hbar \omega)$ are given in Table II for the various strengths of excitation. As can be seen, the frequencies are not constant but decrease with increasing amplitude, indicating some degree of nonlinearity in the response. For comparison, it should be noted that a simple classical estimate of the lowest natural frequency of the slab is given by

$$
\hbar \omega \sim \frac{\hbar \pi}{L} v_{0}=15.3 \mathrm{MeV}
$$

Here $v_{0}=82.7 \mathrm{fm} / 10^{-21} \mathrm{sec}$ is the speed of zero sound derived in Sec. VII, while $L=11.03 \mathrm{fm}$ is the length of the $Q=1.6 \mathrm{fm}^{-2}$ slab. The frequencies of Table II are comparable with the estimate (5.13).

For $\alpha=0.06 \mathrm{fm}^{-2}$, the behavior of the system is quite different, as can be seen from Fig. 4 . The initial expansion is of a much larger amplitude than previously, while afterwards the mean-square length fluctuates about a value considerably greater than that of the ground state. These further fluctuations do not present any well defined beating pattern. For values of $\alpha$ larger than $0.06 \mathrm{fm}^{-2}$ (not shown), the excitation is too strong and the slab splits into two pieces.

In order to determine if the observed beating pattern is due to our very special choice of a linear velocity field [cf., Eq. (5.10)], we have also investigated the response to a cubic velocity field $\left(S \sim z^{4}\right)$. For the $Q=1.6 \mathrm{fm}^{-2}$ slab at an excitation energy corresponding to the $\alpha=0.01 \mathrm{fm}^{-2}$ case above, the same beating behavior of $\left\langle z^{2}\right\rangle$ occurs, with frequencies identical to those of the linear velocity excitation.

Plots of the density distributions $\rho(z, t)$ have not been presented, since they are qualitatively similar to those arising in the collisions displayed in Sec. VI. Qualitatively, they exhibit the expected breathing mode behavior, although the details of the density distribution are by no means periodic in time.

Genuine damping of the slab oscillations is not observed in the cases we have studied. The beating of two modes in the three lowest curves of Fig. 4 is a crude prototype of the damping one would expect as the collective excitation energy is shared between different modes, but with essentially only two degrees of freedom coupled at these low excitation energies and initial conditions, the Poincare cycle time is far too short. The relatively small number of degrees of freedom accessible in the slab geometry is thus crucial to the investigation of dissipation, and this point will be persued further in Sec. VIII below. 
Interaction with an external potential

As a final example of single slab dynamics, we present results for the interaction of a slab with a time-independent external potential barrier. This problem is the simplest which can give rise to the break up phenomena observed in slab-slab reactions. It therefore represents an ideal opportunity for the isolation and study of this feature. Of course, it is also interesting in its own right as an aid in better understanding the behavior of the TDHF solutions. In particular, it furnishes a dramatic example of the collective nature of the slab system.

In this brief presentation of typical results, we use a slab of mass $Q=1.4 \mathrm{fm}^{-2}(N=4$, as shown in Fig. 2). The potential barrier is chosen to be a Gaussian centered about $z=0$ :

$$
V_{\mathrm{ext}}(z)=V_{\mathrm{ext}}^{(0)} \exp \left(-z^{2} / 2 a^{2}\right) .
$$

Here, the width $a$ is chosen to be $2 \mathrm{fm}$. At time $t=0$, the slab is placed so that its interaction with the barrier is negligible and is moving toward $z=0$ with a speed corresponding to $E / A=10 \mathrm{MeV}$ of kinetic energy per nucleon $\left(v=43.8 \mathrm{fm} / 10^{-21}\right.$ sec). To study the penetration process, we may vary the height of the barrier $V_{\mathrm{ext}}^{(0)}$.

Figure 5 shows the time evolution of the density $\rho(z, t)$ for a $10 \mathrm{MeV}$ barrier (i.e., a height equal to the incident kinetic energy). The slab is transmitted with negligible reflection $(<1 \%)$. As can be seen at time $l=0.78 \times 10^{-21} \mathrm{sec}$, when the slab emerges from the barrier its density does not resemble the ground state density at all. Instead, it is in an excited state; the excitation manifesting itself as the motion of density ripples through the transmitted slab. Indeed, $36 \%$ of the incident $\mathrm{ki}-$ netic energy is converted into such excitations.

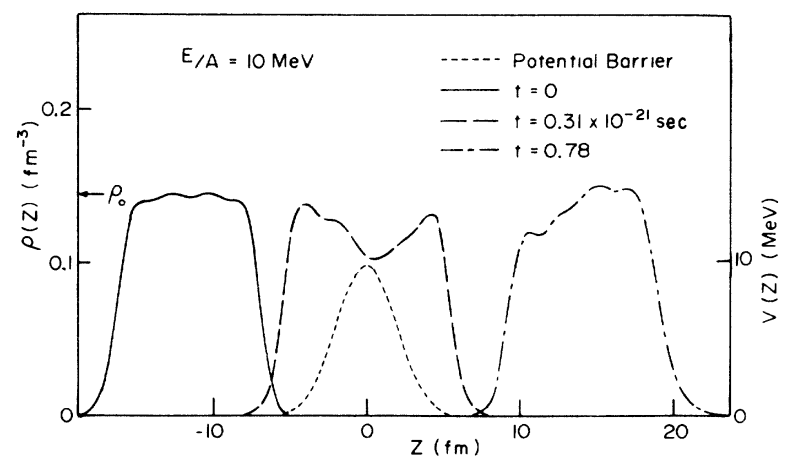

FIG. 5. Density profiles $\rho(z, t)$ at various times $t$ for an $Q=1.4 \mathrm{fm}^{-2}$ slab with bombardment energy $E / A=10$ $\mathrm{MeV}$ interacting with an external potential. The potential barrier, denoted by the short-dashed curve, is referred to the scale at the right, and the scale for the density is given at the left.
Comparison of Fig. 5 with the behavior of a single-particle highlights the collective phenomenon involved. For all practical purposes, we may approximate the Gaussian external potential by an inverted parabolic barrier for which the WKB penetration integral may be done analytically. The penetration of a single nucleon of kinetic energy $E / A$ is then given by

$$
P=\left\{1+\exp \left[2 \pi\left(V_{\mathrm{ext}}^{(0)}-E / A\right) / \hbar \omega\right]\right\}^{-1},
$$

with

$$
\hbar \omega=\left(V_{\mathrm{ext}}^{(0)} \hbar^{2} / m a^{2}\right)^{1 / 2} .
$$

For a kinetic energy equal to the barrier height, Eq. (5.15) gives $P=\frac{1}{2}$, while Fig. 5 indicates that the entire incident slab is transmitted. This is a collective effect, as the attractive single-particle potential created by other nucleons moving through the barrier reduces the height of the effective single-particle barrier.

For a higher barrier $\left(V_{\text {ext }}^{(0)}=20 \mathrm{MeV}\right)$, the time evolution is shown in Fig. 6. Note that here the initial slab splits into slow moving reflected and rapidly moving transmitted pieces, $29 \%$ of the slab being transmitted and $71 \%$ reflected. Again, the final state density fluctuations evidence internal excitation. For this system the collective lowering of the barrier is even more dramatic, for Eqs. (5.15) and (5.16) give a single-particle penetrability of 0.0126 , far less than 0.29 .

From the above results, we can see that the quantity relevant for transmission and reflection is not the single-particle potential barrier, but rather some appropriate collective potential. Such a collective potential is usually considered in an adiabatic limit. For example we may imagine performing a constrained static $\mathrm{HF}$ (CHF) calculation ${ }^{21}$ in the presence of $V_{\text {ext }}$ (although freezing the occupations of the transverse wave functions, of

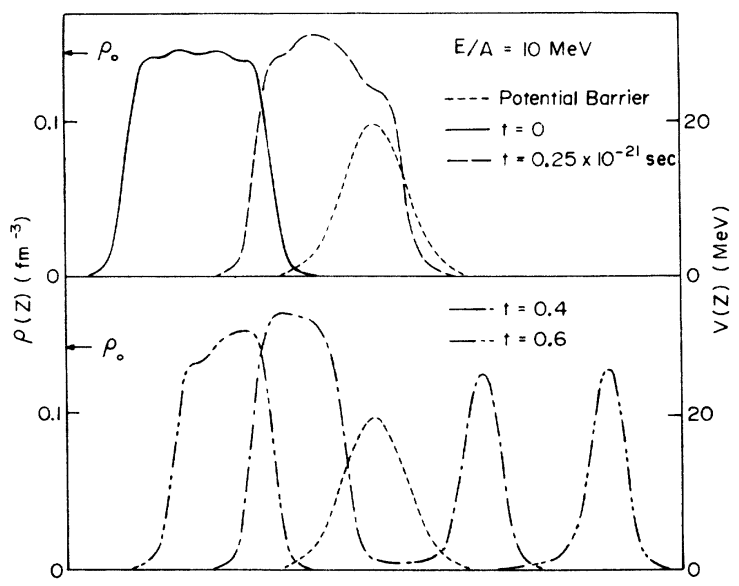

FIG. 6. Density profiles $\rho(z, t)$ as in Fig. 5 . 
course). The physically relevant constraint variable might be $\langle z\rangle$, the position of the center of mass of the slab. The energy of the CHF solution as a function of this variable then defines a collective potential, and some prescription must be adopted for the associated mass parameter. Because the slab has the freedom to minimize its interaction with the external potential in a CHF calculation, we expect the collective potential barrier to be far weaker than the external potential. Since the collective mass parameter should not be drastically different from $A$ times the single nucleon mass, the collective penetrability is thus expected to be considerably larger than the singleparticle value. This discussion is only intended to be qualitative, however, since TDHF has the great conceptual advantages that we never need to choose an ansatz for the constraint parameter, impose an adiabatic approximation, or pick a prescription for inertial parameters.

The collision in Fig. 6, with $V_{\text {ext }}^{(0)}=20 \mathrm{MeV}$, raises a fundamental question of interpretation which will pervade all of the subsequent analysis of collision dynamics. Namely, does all of the slab penetrate the barrier $29 \%$ of the time, does the slab consistently break into two separate slabs such that the transmitted fragment has $29 \%$ of the mass, or do we deal with a complicated superposition of different amplitudes for many different final states? As we shall discuss at length in Sec. VIII, we believe the theory is most meaningful in cases for which the final TDHF fragment represents one single final state with very high probability, in which case it is approximating the most likely reaction channel. This question of interpretation arises in its purest form in these interactions with an external potential, and so this application provides a particularly useful laboratory for future investigation.

In summary, we have briefly presented examples of the interaction of a slab with an external potential barrier. Both total transmission and breakup (transmission and reflection) have been demonstrated. The quantitative details of these features emphasize the collective nature of the TDHF problem.

\section{SLAB COLLISIONS}

This chapter treats the possible reactions occurring between colliding slabs. As will be seen below, given the simplicity of our model, the range of phenomena present is truly remarkable, undoubtedly an indication of the richness of results to be expected in systems of higher dimensionality. For consistency of presentation, we will primarily deal with reactions leading to a compound slab of mass $Q=2.8 \mathrm{fm}^{-2}$. In the symmetric channel, this corresponds to a collision between two $Q=1.4 \mathrm{fm}^{-2}$ slabs, each having $N=4$ occupied orbitals $\phi$. As can be seen from Fig. 2, the $Q=1.4 \mathrm{fm}^{-2}$ slab has a thickness comparable to nuclei in the $\mathrm{Ni}$ region. It is slightly lighter than the lightest slab of the $N=5$ "shell" and therefore is almost magic.

\section{Low energy collisions}

Due to the large amount of information generated in a TDHF calculation, it is useful to have only a few macroscopic coordinates which succinctly summarize the behavior of the system. For a reaction initiated from a symmetric channel, symmetry considerations require that the TDHF density be reflection symmetric with respect to the center of mass (chosen to be $z=0$ ) at all times. A useful set of macroscopic coordinates may then be defined in the following way. ${ }^{22}$ The fragment separation $d$ is taken to be

$$
d(t)=\frac{2}{\mathrm{Q}} \int_{-\infty}^{\infty} \rho(z, t)|z| d z,
$$

where $Q$ is the total mass of the system defined by Eq. (4.21). Note that for separated two fragment shapes (e.g. before a reaction or after scission), $d$ is the distance between the centers of mass of the two pieces. A second coordinate $\sigma$, measuring fragment elongation, is defined as

$$
\sigma=\left(\left\langle z^{2}\right\rangle-\frac{1}{4} d^{2}\right)^{1 / 2},
$$

with

$$
\left\langle z^{2}(t)\right\rangle=\frac{1}{\mathbb{Q}} \int_{-\infty}^{\infty} \rho(z, t) z^{2} d z .
$$

This coordinate is the rms extent of each fragment about its own center of mass. The behavior of the system may then be summarized by the functions $d(t)$ and $\sigma(t)$, or by a trajectory in the $d-\sigma$ plane. Such a trajectory is particularly useful as the gross topography of the potential energy surface in the se coordinates is known, ${ }^{23}$ at least phenomenologically, for three-dimensional collisions. It should be cautioned that while the $d$ and $\sigma$ coordinates reduce to the physically appropriate ones for separated shapes, their meaning is somewhat obscure for fused systems. Nonetheless, they are well defined for these shapes and are useful indicators of dynamics.

We begin with a discussion of reactions initiated by a symmetric $Q_{1}=Q_{2}=1.4 \mathrm{fm}^{-2}$ channel using the initial conditions discussed in Sec. V. The reaction phenomenology will be presented in this section, while a microscopic analysis in terms of the single-particle wave functions is presented in the following section.

To summarize the results, as the bombarding 


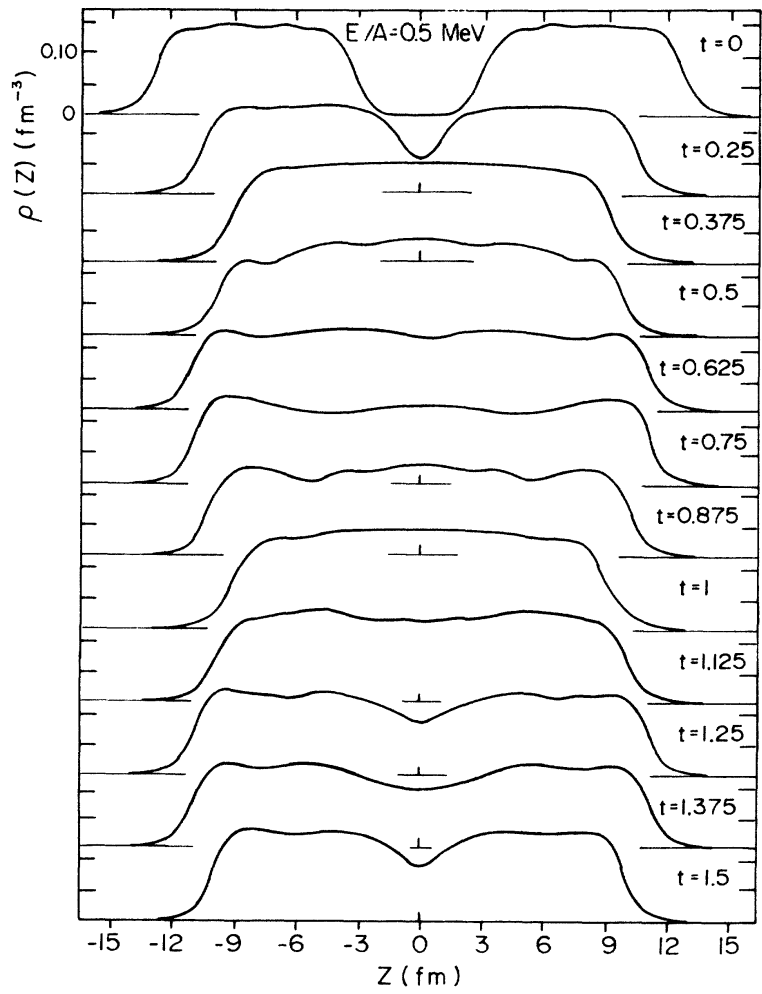

FIG. 7. Density profiles $\rho(z, t)$ at various times $t$ for the reaction $a_{1}=a_{2}=1.4 \mathrm{fm}^{-2}$ at a center-of-mass bombarding energy of $E / A=0.5 \mathrm{MeV}$.

energy per nucleon is increased, the phenomena of fusion, resonances, deep inelastic scattering, and fragmentation are observed.

Figure 7, which shows $\rho(z, t)$ at different times and curve (a) of Fig. 8 , which shows $d(t)$, describe a typical fusion reaction at $E / A=0.5 \mathrm{MeV}$. Recall

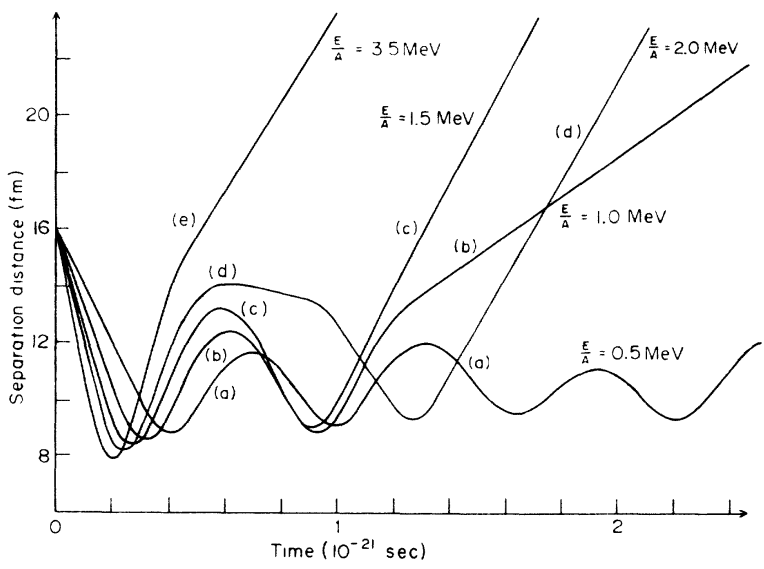

FIG. 8. The fragment separation coordinate $d(t)$ for the $a_{1}=a_{2}=1.4 \mathrm{fm}^{-2}$ reaction at various bombarding energies.

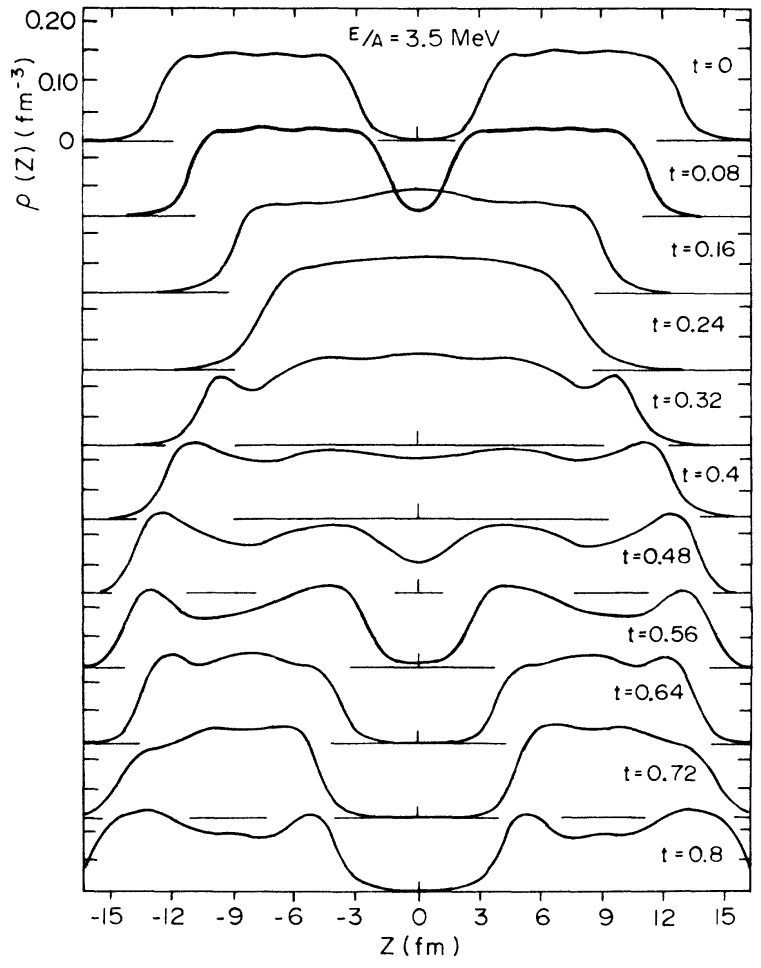

FIG. 9. Density profiles $\rho(z, t)$ as in Fig. 7 for $E / A$ $=3.5 \mathrm{MeV}$.

that a straight line on the latter figure corresponds to motion of the fragments with a constant relative velocity, as in the initial part of all curves $d(t)$ before the fragments collide. After an initial fusion and maximum compression, at $t \sim 0.4 \times 10^{-21}$ sec, the compound system continues to oscillate without reaching a scission configuration. The period of these oscillations, $0.57 \times 10^{-21} \mathrm{sec}$, is comparable with the simple classical estimate $T=2 L / v_{0}=0.47 \times 10^{-21} \mathrm{sec}$, where $L=19.31 \mathrm{fm}$ is the thickness of the compound slab and $v_{0}$ $=82.7 \mathrm{fm} / 10^{-21} \mathrm{sec}$ is the zero sound speed derived in Sec. VII. These oscillations persist for as long as we have integrated the TDHF equations ( $\sim 5$ cycles or to $\left.t \sim 3 \times 10^{-21} \mathrm{sec}\right)$. This type of behavior is just what is to be expected of a time-dependent description of a compound nucleus in which the excitation energy, initially in the coherent form of uniform translation of the target and projectile, has been degraded into internal excitation of the fused system. ${ }^{24}$ As discussed in the previous section, in a realistic system with more degrees of freedom, the shape oscillations exhibited by our calculation would be expected to damp after several cycles because of further degradation of the energy. Of course, if the TDHF equations could be integrated for a time comparable 
with the Poincaré time of the system $\left(z 10^{-18}\right.$ for a real nucleus), we would eventually see the energy focused back into a fragment separation mode, resulting in scission.

We now turn to an intermediate energy collision $E / A=3.5 \mathrm{MeV}$ illustrated by Fig. 9 and curve (e) of Fig. 8. This reaction typifies a deep inelastic process in which the system separates directly after fusion. The scission dynamics appear to proceed along the lines expected from fluid dynamics. After the initial compression, the subsequent relaxation stretches the system to a more or less uniform density $\sim \frac{2}{3} \rho_{0}$ at time $t=0.4 \times 10^{-21}$ sec. At this time, density oscillations appear and grow until the scission. Note that the scission configuration coincides with the bend in curve (e) at $t \simeq 0.5 \times 10^{-21} \mathrm{sec}$ indicating the comparative suddenness of the separation. After separation, the fragments move apart with a constant relative velocity [the final straight portion of curve (e)], which is smaller than the initial velocity. Some of the initial kinetic energy has been converted into internal excitation of the fragments. One manifestation of this excitation is the density fluctuations sloshing back and forth in the fragments after time $t \sim 0.56 \times 10^{-21} \mathrm{sec}$. In this particular collision $\sim 90 \%$ of the initial kinetic energy has been lost to internal excitation.

It is interesting to note the beginning of a shock phenomenon in the initial stages of this reaction, at $t \sim 0.16 \times 10^{-21} \mathrm{sec}$ (see Fig. 9). Such a density discontinuity must, of course, occur in the initial stages of all our one-dimensional calculations, although it is very weak at low bombarding energies $(E / A \lesssim 3 \mathrm{MeV})$. A detailed discussion of the shock phenomena occurring in our model is given below.

The reaction dynamics between $E / A=0.5$ and 3 $\mathrm{MeV}$ are summarized in Fig. 10 which shows the percentage of kinetic energy remaining in relative

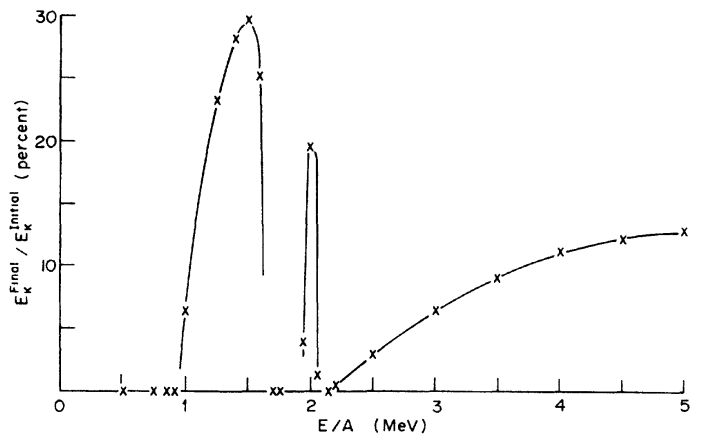

FIG. 10. The coefficient of restitution $c_{r}$ defined by Eq. (6.4) as a function of $E / A$ for the $1.4 \mathrm{fm}^{-2}$ plus 1.4 $\mathrm{fm}^{-2}$ system. Calculated points are denoted by a cross. $A$ value of zero denotes fusion. motion after the collision (coefficient of restitution) given by

$$
C_{r} \equiv \frac{E_{k}^{\text {Final }}}{E_{k}^{\text {Initial }}}=\left(\frac{\dot{d}(t=+\infty)}{\dot{d}(t=0)}\right)^{2} .
$$

The final relative velocity $\dot{d}(t=+\infty)$ is easily evalu ated from the asymptotic slope of the $d(t)$ curve in Fig. 8. A value of zero for $C_{r}$ indicates fusion.

The two peaks at $E / A \sim 1.3 \mathrm{MeV}$ and $E / A \sim 2.0$ $\mathrm{MeV}$ indicate resonant processes in which the system undergoes fission after one oscillation of the compound system. Curves (b) and (c) of Fig. 8 and Fig. 11 indicate these processes. Note that the second compression in these reactions [i.e., minimum in $d(t)]$ is either resonant or antiresonant with the oscillations of the fused system. This phenomenon can be understood both in macroscopic terms, which are adopted here, and microscopic terms treated subsequently. The resonance conditions are further illustrated by Fig. 12, in which the trajectories in the $d-\sigma$ plane are shown for various bombarding energies.

At low energies, in the resonance region, too much energy is concentrated in the $\sigma$ and other degrees of freedom for scission to take place at

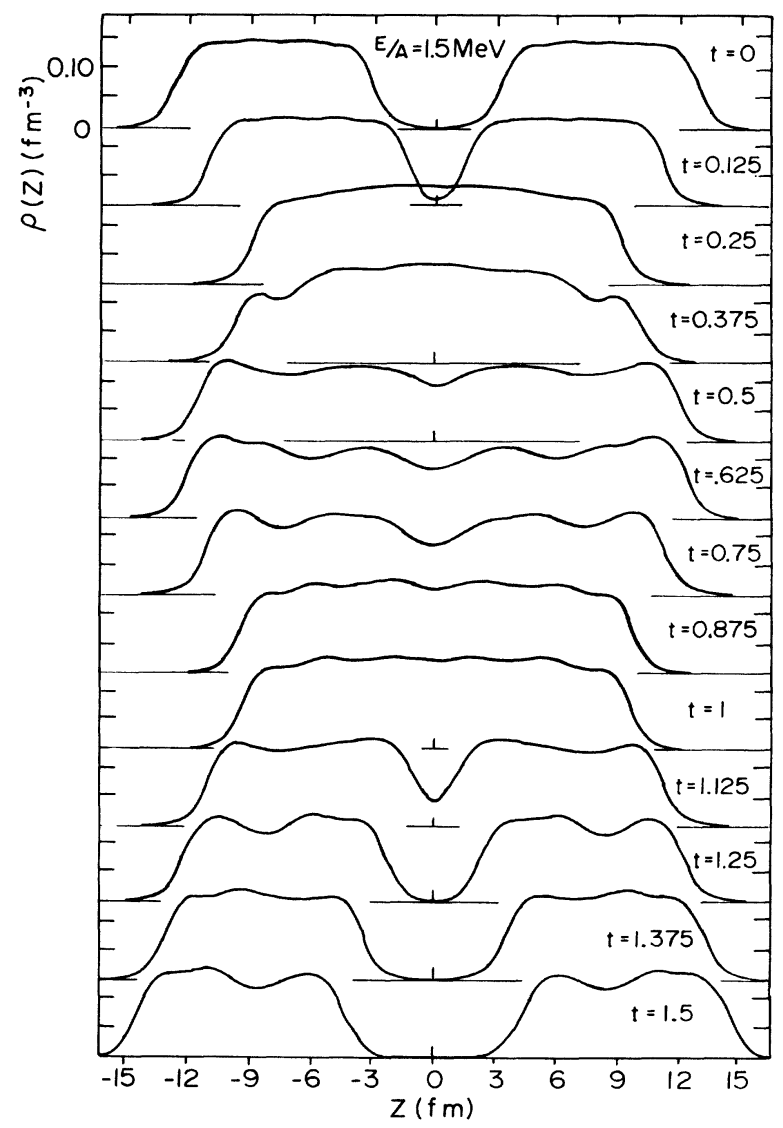

FIG. 11. Density profiles $\rho(z, t)$ as in Fig. 7 . 


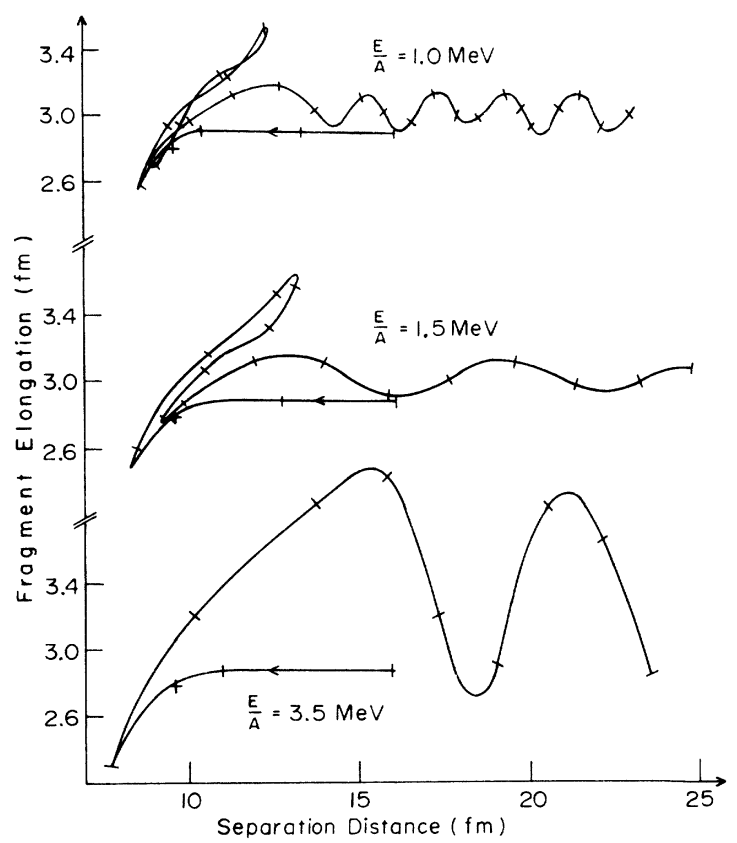

FIG. 12. Trajectories in the separation-elongation $(d-\sigma)$ plane for the $1.4 \mathrm{fm}^{-2}$ plus $1.4 \mathrm{fm}^{-2}$ system at various bombarding energies. Hash marks are at intervals of $0.1 \times 10^{-21} \mathrm{sec}$.

the first oscillation. Since a large value of $\sigma$ corresponds to a highly elongated fragment, this configuration requires a significant amount of energy because the density is consistently below saturation density for the entire fragment. At higher energy, such as $E / A=3.5 \mathrm{MeV}$, enough energy remains in the $d$ degree of freedom for immediate scission. In contrast, for $E / A=1.5$ or $2 \mathrm{MeV}$, since insufficient energy is available in the $d$ degree of freedom, the system does not separate. During subsequent oscillations, energy is transferred between $\sigma$ to $d$, so that scission conditions may occur at a later time. Thus, the resonance depends crucially upon the interplay of the fragment elongation and fragment separation modes. After scission, the fragments continue to oscillate in the two-fragment "valley" of the potential energy surface as they move apart. ${ }^{23}$

On the basis of the calculations we have performed, we can not rule out the presence of other, "narrower" resonances in Fig. 10, at energies $E / A \lesssim 2.5 \mathrm{MeV}$. Indeed, reference to curve (d) of Fig. 8 shows that such peaks are expected. The very slow relative motion at the maximum of the first elongation $\left(t \sim 0.5-0.8 \times 10^{-21} \mathrm{sec}\right)$ indicates that the relaxation of the first compression almost succeeds in fission. However, the fragments stop moving apart $\left(t \sim 0.5 \times 10^{-21} \mathrm{sec}\right)$ and very slowly begin to fall back toward one another, finally re- sulting in the second compression. This recapture time is the factor which determines resonance. Thus, we might expect to see other resonances, corresponding to the second compression occurring after several oscillations of the fragments with consequently longer recapture times. As the recapture time must depend very sensitively upon the configuration at the first elongation, these peaks in $C_{r}$ are expected to be extremely narrow. Indeed, the $E / A=2 \mathrm{MeV}$ peak is significantly nar rower than the $1.3 \mathrm{MeV}$ peak, indicating the extreme sensitivity of these processes to the initial conditions.

Figure 13 shows a reaction leading to the same compound system $Q=2.8 \mathrm{fm}^{-2}$, initiated from the asymmetric channel $Q_{1}=1.9 \mathrm{fm}^{-2}+a_{2}=0.9 \mathrm{fm}^{-2}$ at $E / A=4 \mathrm{MeV}$. Note that when formed from the

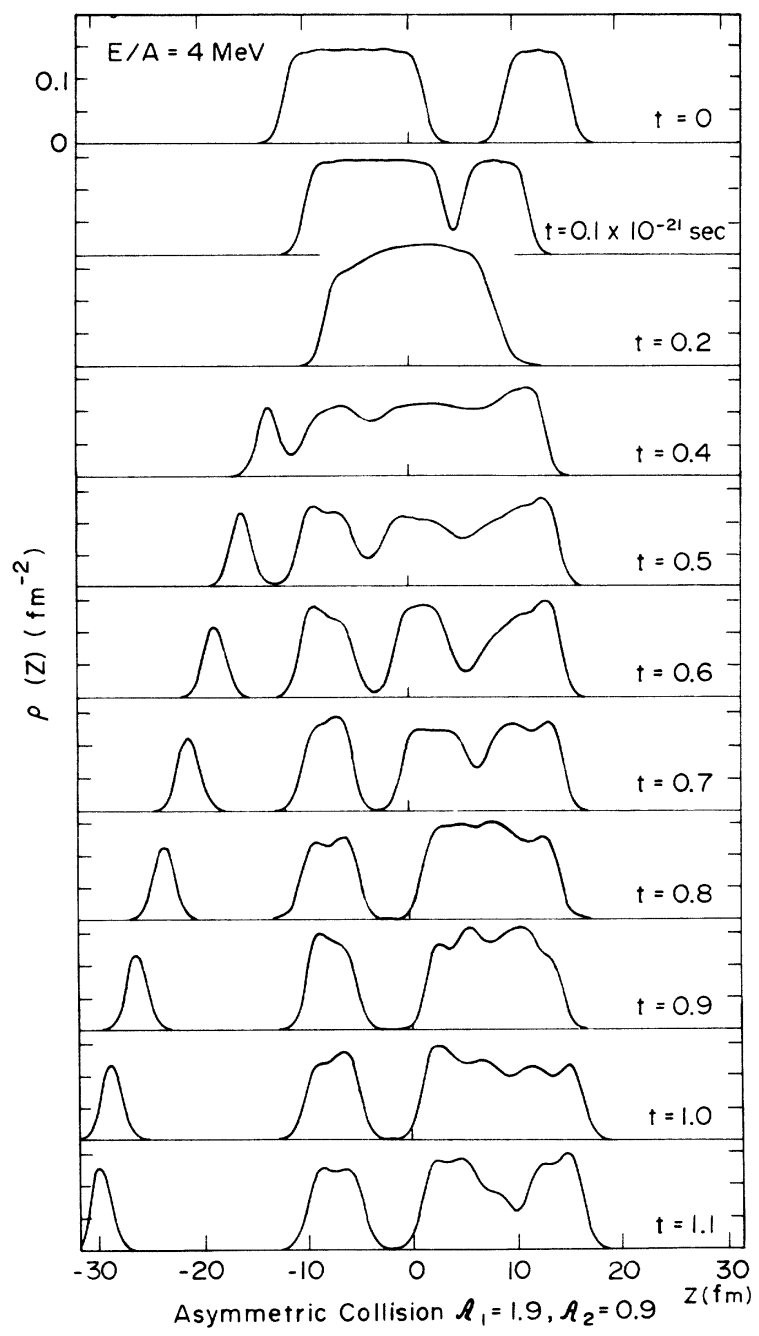

FIG. 13. Density profiles $\rho(z, t)$ at various times $t$ for the reaction $Q_{1}=1.9 \mathrm{fm}^{-2}+Q_{2}=0.9 \mathrm{fm}^{-2}$ at $E / A=4 \mathrm{MeV}$. 
symmetric channel at this excitation energy, Fig. 10 indicates that the compound system fissions into two fragments in a deep inelastic event, with $C_{r} \simeq 11 \%$. However, the asymmetric reaction results in fragmentation of both target and projectile, the final state consisting of three distinct slabs in various states of excitation. This fragmentation is typical of the symmetric channel above $E / A \sim 6$ $\mathrm{MeV}$, and is discussed below.

\section{High energy collision phenomenology}

The two most salient features of high energy collisions are fragmentation and propagation of shock waves. Both features are evident in the sequence of density distributions presented in Figs. 14(a) and 14 (b) for the case $E / A=25 \mathrm{MeV}$. (Recall that c.m. energies are always specified.) The evolution of the reaction begins with an increase in central density which propagates outward in a manner suggestive of a shock wave. A distinct lump then appears at the nuclear surface and, unlike the similar lumps at lower energies (cf. Fig. 9 at $t=0.32$ $\times 10^{-21} \mathrm{sec}$ ) actually separates off from the rest

(a)

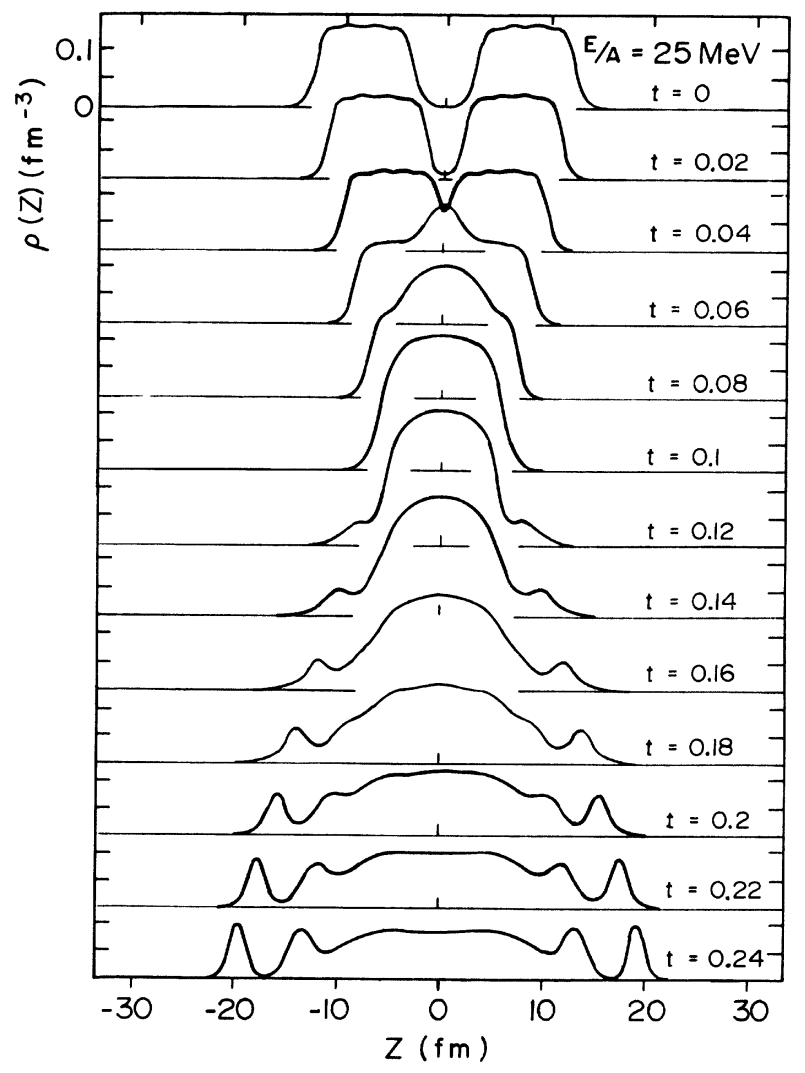

of the system, traveling faster than the velocity of the incident projectile. If we consider two hypothetical markers originally aligned with the leading edges of the colliding slabs and moving with the initial velocity, we observe that the remaining matter is spread out between these two markers at subsequent times. Initially the matter is rather smoothly distributed between the two markers, with a density considerably lower than the saturation density $\rho_{0}=0.145 \mathrm{fm}^{-3}$. As the final state evolves, however, individual fragments condense out of this low density region, beginning at the exterior edges and continuing throughout the interior region.

The systematics of fragmentation are indicated in Fig. 15, which shows one-half of the final density distribution for four collisions ranging from $E / A=10$ to $50 \mathrm{MeV}$. To make the final states comparable, we have considered the position the leading edge marker of the right slab would have reached under free propagation at the original velocity. The final states are selected at times such that this marker is at approximately $-25 \mathrm{fm}$. At $E / A=10 \mathrm{MeV}$ the leading particle appears to have insufficient energy to break off, whereas $E / A=16 \mathrm{MeV}$ is a transition case. At higher en-

(b)

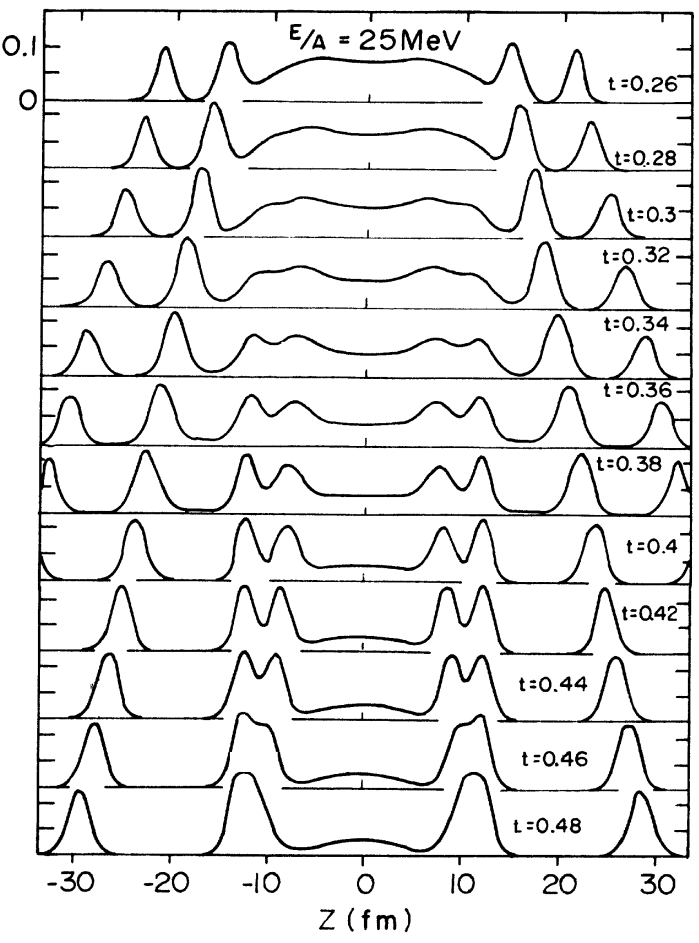

FIG. 14. (a), (b) Density profiles $\rho(z, t)$ as in Fig. 7. 


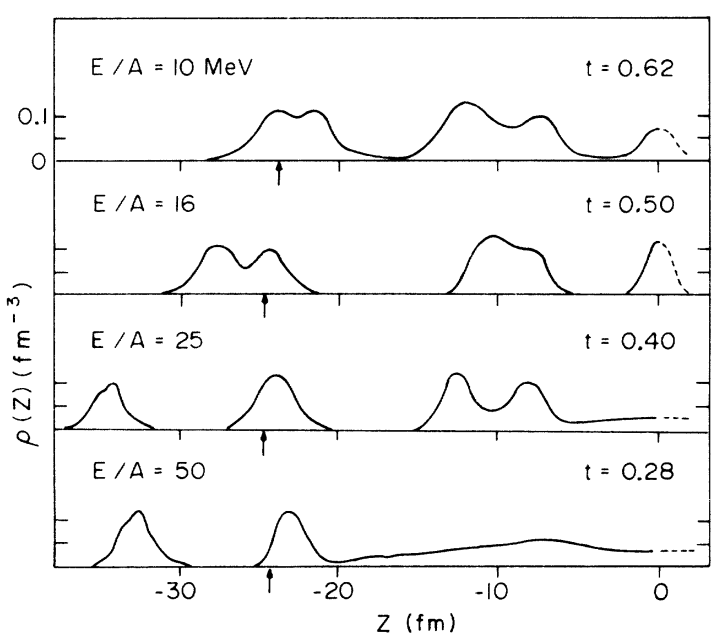

FIG. 15. Final state density profiles for the $a_{1}=a_{2}$ $=1.4 \mathrm{fm}^{-2}$ system at various bombarding energies. The arrow at $z=-25 \mathrm{fm}$ indicates the position of the marker used to define equivalent times, as discussed in the text.

ergies separation actually occurs. As the energy increases, the size of the region over which condensation into fragments occurs also increases. In the case $E / A=10 \mathrm{MeV}$ condensation into distinct fragments is complete by the time the marker is at $25 \mathrm{fm}$. At $E / A=25 \mathrm{MeV}$ condensation in the interior region is not yet complete, and at $E / A=50$ $\mathrm{MeV}$ the density within $20 \mathrm{fm}$ of the origin is still almost uniform.

These fragmentation results are particularly tantalizing in view of the picture which is emerging from high energy hadron-nucleus scattering. From recent experiments, it appears that hadrons must be regarded as composite many-body systems. Furthermore, in collisions these composite particles appear to require substantial times and distances to form their final states. ${ }^{25}$ Therefore we shall attempt to understand the microscopic origin of our final states in Sec. VII and will critically examine the physical limitations of the onedimensional geometry in Sec. VIII.

In the context of conventional fluid dynamics, a shock wave is an interface at which fluid variables such as density and velocity change discontinuously. The propagation of this shock front is straightforwardly specified by conservation laws and the equation of state of the fluid. ${ }^{26,27}$ On a microscopic scale, of course, the interface is not discontinuous, and the scale of the transition region is set by the mean free path of the constituent particles. Sobel et $a l .{ }^{28}$ indicate that the transition region is certainly larger than the longitudinal momentum decay length of $3.5 \mathrm{fm}$, and Bertsch ${ }^{29}$ argues that the thickness should be of the order of 2.5 times the mean free path, or roughly $8 \mathrm{fm}$.
High energy collisions of slabs exhibit similar behavior which we loosely refer to as shock waves in spite of the fact that we are not dealing with a conventional fluid dynamics. To emphasize such shock behavior, we have calculated collisions for thick slabs with $Q_{1}=Q_{2}=2.4 \mathrm{fm}^{-2}$. A typical case, for $E / A=10 \mathrm{MeV}$, is shown in Fig. 16. As the slabs interpenetrate, the central density first increases and then stabilizes at some constant value. The rather distinct interfaces between the interior and exterior densities then propagate outward until they reach the surface.

For the shock wave picture to be very meaningful, the conditions of steady state flow must be approximately satisfied. In steady state flow for symmetric collisions, the mean velocity of the interior nuclear matter should be zero and the velocity of the exterior matter at density $\rho_{0}$ should be the incident velocity. By symmetry, the center of the slab is a stagnation point. One simple indication of the degree to which steady state is ob-

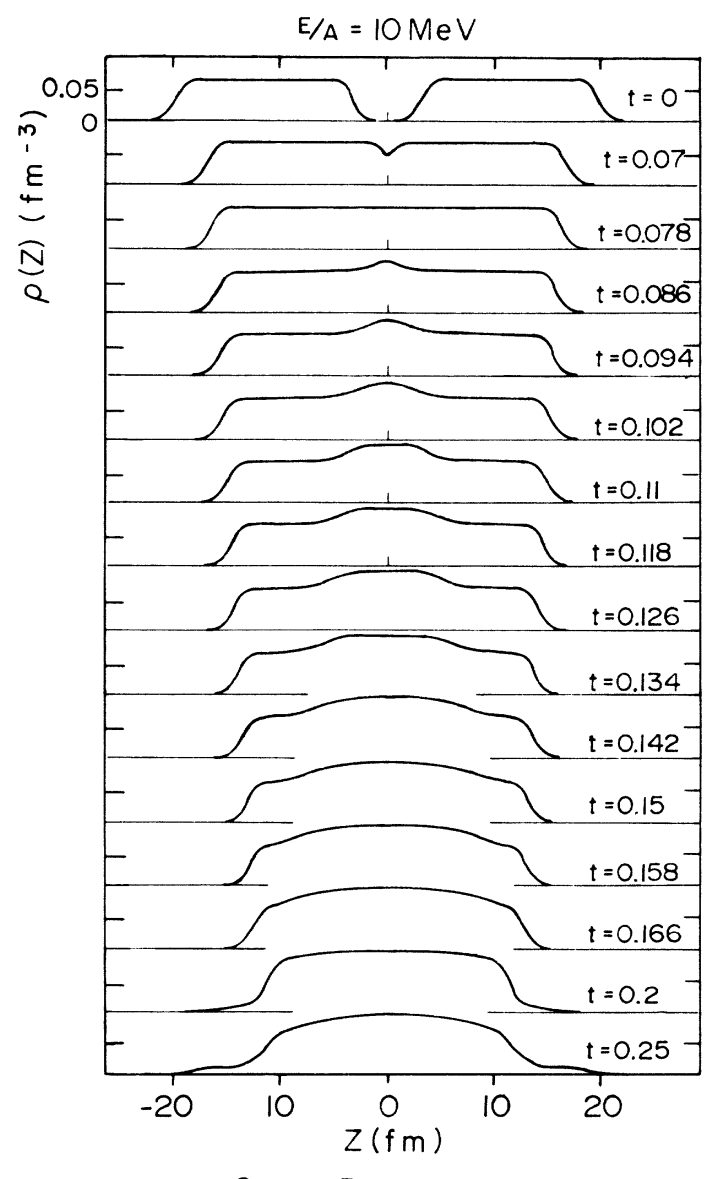

SHOCK PROPAGATION

FIG. 16. Density profiles $\rho(z, t)$ at various times $t$ for the $Q_{1}=Q_{2}=2.4 \mathrm{fm}^{-2}$ system at $E / A:: 10 \mathrm{MeV}$. 
tained is provided by the behavior of the central density as a function of time which is shown in Fig. 17. The times at which the shock is first clearly evident at the center $\left(t_{1}\right)$, the shock has traveled halfway across the slab $\left(t_{2}\right)$ and the shock has reached the edge of the slab $\left(t_{3}\right)$ are indicated in the figure. Although the central density is approximately constant at $E / A=10 \mathrm{MeV}$, it changes significantly during the transit time of the shock at $E / A=25 \mathrm{MeV}$.

Assuming that the interior density $\rho$ and the exterior density $\rho_{0}$ remain constant in time, the continuity equation yields the velocity $s$ at which the shock front propagates ${ }^{27}$ :

$$
s=\frac{v_{i}}{\left(\rho / \rho_{0}-1\right)},
$$

where $v_{i}$ is the initial velocity. Because of the difficulty of uniquely defining the center of the broad shock front, Eq. (6.5) is the only quantitative definition we have found for the shock speed. Even it is not unique, because the interior density $\rho$ is not exactly constant in space or time. Hence, we have adopted the convention of defining $s$ using the central density at time $t_{2}$ in Eq. (6.5), which yields the results shown in Table III. The shock speed $s$ is expected to be greater than or equal to the zero sound speed, $82.7 \mathrm{fm} / 10^{-21} \mathrm{sec}$, derived in Sec. VII. This is observed in Table II. In addition, at very low energies $(E / A \lesssim 5 \mathrm{MeV})$ the shock kinematics are in agreement with steady state predictions. ${ }^{30}$ In the high energy limit, in which the slabs essentially pass through each other with relatively minor effects of interactions, during the early stages of the collision the shock wave description simply degenerates into the superposition of two slabs moving at their initial velocities. The interior density is then twice $\rho_{0}$, yielding a shock speed equal to the initial velocity $v_{i}$. The case $E / A=50 \mathrm{MeV}$ is already rather close to this limit. To verify that nothing unexpected occurs at very high energies, we have pushed the nonrelativistic TDHF approximation well beyond its region of validity to calculate a collision at $E / A=117.5 \mathrm{MeV}$. This case corresponds to a relative velocity equal to the speed of light. The central density at time $t_{2}$ is $1.98 \rho_{0}$ and the "shock" thus propagates at the velocity of the initial slabs. Therefore, at least in the context of the present model, our simple interpretation holds at high energy and no exotic high density states are created by shock waves.

To display the structure of the transition region in greater detail, Fig. 18 shows profiles of the density $\rho(z)$, and the velocity field $v(z)$, defined by Eq. (5.2a), plotted in a frame moving with the shock speed $s$ for the cases tabulated in Table III. The short-dashed, solid, and long-dashed curves denote the profiles at time $t_{1}, t_{2}$, and $t_{3}$, respectively. In all cases the initial slabs correspond to $Q_{1}=Q_{2}=2.4 \mathrm{fm}^{-2}$. For the collisions at $E / A=10$ $\mathrm{MeV}$, steady state flow is a good approximation and the transition region maintains its shape with a thickness of approximately $4 \mathrm{fm}$. At higher energy, however, steady state flow does not occur, and the transition region degrades significantly. The final thickness of the transition region at $E / A$ $=50 \mathrm{MeV}$, for example, is roughly $7 \mathrm{fm}$.

Certainly application of these results to finite nuclei requires great caution, because of the artificial features of slab geometry. Whereas these one-dimensional collisions are an ideal theoretical laboratory for investigating the relation between TDHF and fluid dynamics, for systems as small as the nuclei found in nature the ejection of matter in the transverse direction is an essential element. Thus such features as the continued increase in density shown in Fig. 18 after the passage of the shock are most probably irrelevant to finite nuclei. However, conclusions concerning the structure of the transition region and the high energy behavior do appear to bear some relevance. It is quite plausible that a two-dimensional application will still give rise to transition regions of thicknesses of the order of 4 to $7 \mathrm{fm}$. If there is any change in this transition region, we would expect it to degrade further with the addition of transverse degrees of freedom. Hence, the extreme fluid dynamic assumption of local equilibration giving rise to discontinuous shock fronts may be exceedingly misleading when the actual transition regions are of the order of the radii of the nuclei involved. ${ }^{31-33}$ Furthermore, the intuitively attractive notion of

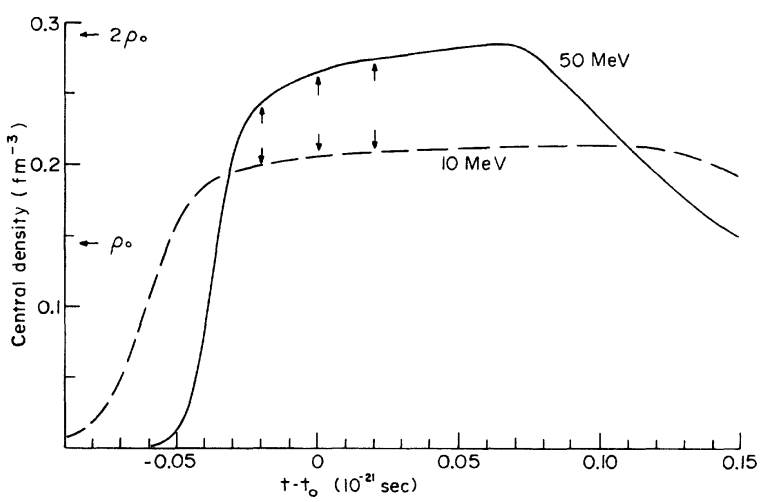

FIG. 17. Central density as a function of time for the $a_{1}=a_{2}=2.4 \mathrm{fm}^{-2}$ system at two energies. The origin of the time scale has been shifted by an arbitrary constant $t_{0}$ and the arrows denote the times $t_{1}, t_{2}$, and $t_{3}$ described in the text. 
TABLE III. Fractional increase in central density, incident slab velocity $v_{i}$, and shock velocity $s$ defined by Eq. (6.5) for the three cases displayed in Fig. 18.

\begin{tabular}{cccc}
\hline$\frac{\rho}{A}(\mathrm{MeV})$ & $\frac{\rho\left(\boldsymbol{t}_{2}\right)}{\rho_{0}}$ & $v_{i}\left(\mathrm{fm} / 10^{-21} \mathrm{sec}\right)$ & $s\left(\mathrm{fm} / 10^{-21} \mathrm{sec}\right)$ \\
\hline 10 & 1.42 & 44 & 105 \\
25 & 1.65 & 69 & 107 \\
50 & 1.83 & 98 & 118 \\
\hline \hline
\end{tabular}

simple interpenetration at high energies appears to be borne out by the present TDHF approximation and is in marked contrast to attainment of densities much greater than $2 \rho_{0}$ obtained with a fluid dynamic ansatz. We must therefore conclude that if reactions in this regime are amenable to a fluid dynamic treatment at all, the rheology of this fluid is very different than usually postulated.

\section{MICROSCOPIC ASPECTS OF TDHF DYNAMICS}

One of the primary limitations of a theory such as TDHF which requires the numerical solution of a set of nonlinear equations is the problem of obtaining physical insight into the structure of the resulting solutions. In this section, we shall examine some of the essential features of the results for our one-dimensional model and attempt to assess their physical origin and significance.

Special features of one-dimensional geometry

At the outset, before examining specific computational results, it is useful to isolate certain features which are directly related to the specialization to one-dimensional geometry. We first consider the effect of this geometry on two simple sound modes: thermodynamic sound and zero sound. Since the one-body density matrix contains the complete information concerning our manybody wave function, we shall be interested in examining its off-diagonal behavior in coordinate space. Hence, we shall explore in this section the effect of our geometry on the density matrix of an infinite Fermi gas. Finally, for subsequent examination of single-particle effects, we shall discuss the special role of single-particle wave functions in our slab geometry.

We first consider the sound modes. Ordinary thermodynamic sound arises under conditions such that three-dimensional local equilibration occurs. ${ }^{34}$ Thus, we may determine $E / A$ assuming that plane wave states are isotropically populated for all $k<k_{F}=\left(3 \pi^{2} \rho / 2\right)^{1 / 3}$ and derive the speed of thermodynamic sound from the relation:

$$
v_{\mathrm{th}}^{2}=\left.\frac{\rho^{2}}{m} \frac{\partial^{2} E / A}{\partial \rho^{2}}\right|_{\rho_{\mathrm{o}}}=\frac{K}{9 m},
$$

where $K$ is defined in Eq. (4.10). For our interaction described in Sec. III, $v_{\text {th }}=62.6 \mathrm{fm} / 10^{-21} \mathrm{sec}$.

In slab geometry, however, due to the decoupling of the transverse wave functions $(1 / \sqrt{\Omega}) e^{i \overrightarrow{\mathrm{k}}_{\perp} \cdot \overrightarrow{\mathrm{r}}_{\perp}}$ from the wave functions $\phi_{n}(z)$, equilibration between the $z$ direction and the two transverse directions cannot occur. Therefore, each function $\phi_{n}(z)$ is associated with the same transverse wave functions, irrespective of the density, and the Fermi sphere distorts in momentum space as the density is varied instead of remaining spherical and simply changing radius.

Consider, now, a large cubic box of side $L$ with periodic boundary conditions filled with plane wave states in all three dimensions up to the equilibrium Fermi momentum $k_{F}^{0}$ corresponding to the saturation density $\rho_{0}$. If the transverse wave functions are decoupled from the $z$ wave functions and the length of the box in the $z$ direction is then varied, the functions $\phi_{k}(z)$ become

$$
\phi_{k}(z) \Rightarrow\left(\frac{\rho}{\rho_{0} L}\right)^{1 / 2} e^{i\left(\rho_{k} z / \rho_{0}\right)} .
$$

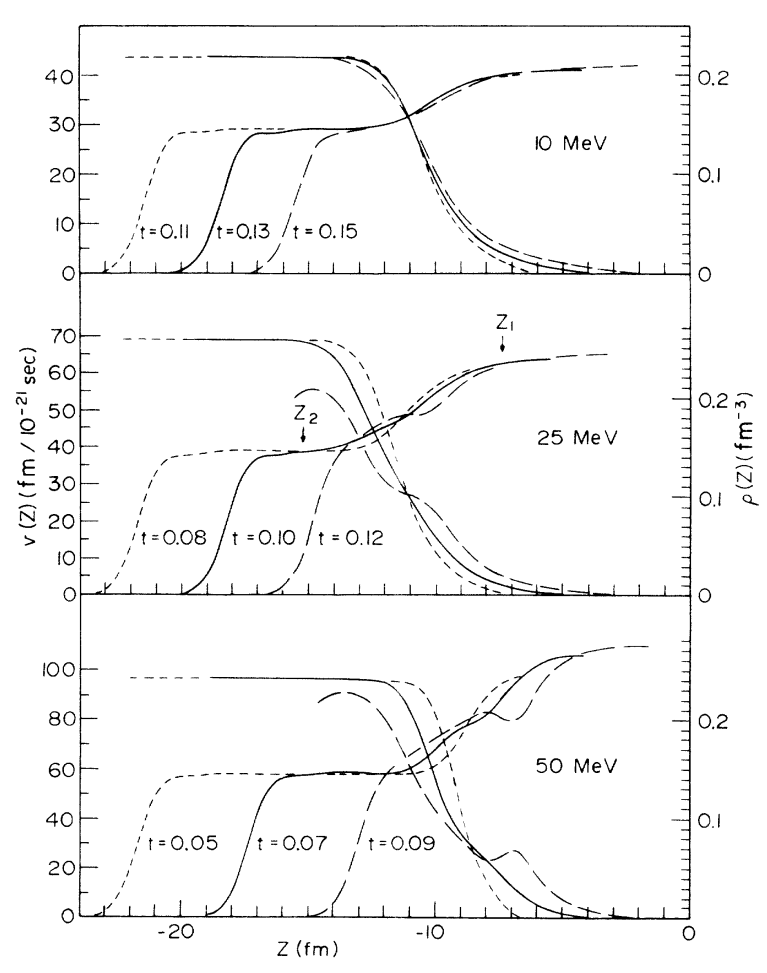

FIG. 18. Slab density profiles and velocity fields for the $Q_{1}=Q_{2}=2.4 \mathrm{fm}^{-2}$ system in the shock frame defined by Eq. (6.5). The short-dashed, solid, and long-dashed lines correspond to the times $t_{1}, t_{2}$, and $t_{3}$ defined in the text. The velocity scale is given on the left ordinate and the density scale is given on the right. Note the arrows denoting the positions $z_{2}$ and $z_{3}$ referred to in Fig. 21. 
Note that this transformation corresponds to deforming the unperturbed Fermi sphere into an ellipsoid. Hence, instead of Eq. (4.8), the appropriate expression for $E / A$ as a function of $\rho$ with the transverse wave functions frozen is

$$
\begin{aligned}
\left.\frac{E}{A}\right|_{1-D}= & \frac{1}{10} \frac{\hbar^{2}}{m}\left(\frac{3 \pi^{2}}{2}\right)^{2 / 3} \rho_{0}{ }^{2 / 3}\left[\left(\frac{\rho}{\rho_{0}}\right)^{2}+2\right] \\
& +\frac{3}{8}\left(t_{0}+2 \pi a^{3} V_{0}\right) \rho+\frac{1}{16} t_{3} \rho^{2} .
\end{aligned}
$$

The speed of one-dimensional thermodynamic sound is then given by

$$
\begin{aligned}
v_{1-D}^{2} & =\left(\left.\frac{\rho^{2}}{m} \frac{\partial^{2} E / A}{\partial^{2} \rho}\right|_{1-D}\right)_{\rho_{0}} \\
& =\frac{1}{m}\left(\frac{K}{9}+\frac{\hbar^{2} 4 k_{F}^{2}}{15 m}\right) .
\end{aligned}
$$

This agrees with the long-wavelength limit of the general expression derived by Koonin ${ }^{27}$ and yields $v_{1-D}=75.3 \mathrm{fm} / 10^{-21} \mathrm{sec}$.

The 1-dimensional thermodynamic sound discussed above assumes complete equilibration in the $z$ direction, which physically arises from twobody collisions. The TDHF equations, however, incorporate two-body collisions only to the extent to which they contribute to the mean field. In the long-wavelength limit the TDHF equation for the density matrix is equivalent to a collisionless Boltzmann equation, so the relevant sound mode is not thermodynamic sound but rather zero sound. ${ }^{35}$ For the case of slab geometry zero sound propagates along the $z$ axis, corresponding to infinitesimal axially symmetric distortions of Fermi sphere which are not reflection symmetric with respect to the $x-y$ plane.
The velocity of zero sound is most easily estimated by noting that the interaction developed in Sec. III is very similar to Skyrme VI and using the tabulated Landau parameters from Ref. 36 . Since $m^{*}=m, F_{1}=0$ and the speed of zero sound $v_{0}$ is determined from ${ }^{35}$

$$
\frac{1}{2} r \ln \left(\frac{r+1}{r-1}\right)-1=\frac{1}{F_{0}},
$$

where $r=v_{0} / v_{F}$ and $v_{F}$ is the Fermi velocity. Using the value $F_{0}=0.74$ for Skyrme VI yields $r$ $=1.020$ and thus $v_{0}=82.7 \mathrm{fm} / 10^{-21} \mathrm{sec}$.

Since $r$ derived from Eq. (7.5) is very insensitive to $F_{0}$ and must be greater than 1 , it is safe to conclude that $v_{0}$ is a few percent larger than $v_{F}=81.1 \mathrm{fm} / 10^{-21} \mathrm{sec}$ for our interaction.

The off-diagonal structure of the one-body density matrix $\rho\left(\vec{r}, \vec{r}^{\prime}\right)$ is also significantly influenced by the 1-dimensional geometry. For a three-dimensional Fermi gas, the density matrix is isotropic in the relative coordinate $\overrightarrow{\mathbf{s}}=\overrightarrow{\mathbf{r}}-\overrightarrow{\mathbf{r}}^{\prime}$ :

$$
\rho(\overrightarrow{\mathrm{R}}+\overrightarrow{\mathrm{s}} / 2, \overrightarrow{\mathrm{R}}-\overrightarrow{\mathrm{s}} / 2)=\rho\left[\rho_{\mathrm{SL}}\left(k_{F} s\right)\right]
$$

where the Slater density is defined by

$$
\rho_{\mathrm{SL}}\left(k_{F} s\right)=\frac{3 j_{1}\left(k_{F} s\right)}{k_{F} s}
$$

and $j_{1}$ is the usual spherical Bessel function.

Because the range of nonlocality scales with $k_{F}$, it varies as $\rho^{-1 / 3}$. In contrast, when the trans verse wave functions are frozen into the states appropriate for $\rho_{0}$, we must return to the case of a box with a variable length in the $z$ direction and use the wave functions in Eq. (7.2). The general expression is then

$$
\rho(\overrightarrow{\mathrm{R}}+\overrightarrow{\mathrm{S}} / 2, \overrightarrow{\mathrm{R}}-\overrightarrow{\mathrm{S}} / 2)=\frac{\rho}{\pi^{2} \rho_{0}} \int_{-k_{F}^{0}}^{k_{F}^{0}} \exp \left[i\left(\rho / \rho_{0}\right) k_{z} s_{z}\right] \int_{0}^{\left[\left(k_{F}^{0}\right)^{2}-k_{z}^{2}\right]^{1 / 2}} \exp \left(i k_{\perp} s_{\perp}\right) k_{\perp} d k_{\perp} d k_{z} .
$$

In the transverse direction, the range of nonlocality is frozen at the value specified by $k_{F}^{0}$ :

$$
\rho_{1-D}\left(s_{\perp}\right)=\rho \frac{3 j_{1}\left(k_{F}^{0} s_{\perp}\right)}{\left(k_{F}^{0} s_{\perp}\right)}
$$

In the $z$ direction, the off-diagonal density matrix is given by

$$
\rho_{1-D}\left(s_{z}\right)=\rho \frac{3 j_{1}\left[\left(\rho / \rho_{0}\right) k_{F}^{0} s_{z}\right]}{\left(\rho / \rho_{0}\right) k_{F}^{0} s_{z}} .
$$

Thus, the range of nonlocality varies with $\rho^{-1}$ for one-dimensional geometry, in contrast to the three-dimensional variation with $\rho^{-1 / 3}$. This anomalous density dependence will be evident in our results.
For subsequent comparison, it would also be useful to define Fermi gas density matrices at finite temperature for both three and one-dimensional geometry. ${ }^{37}$ In three dimensions,

$$
\rho_{T}(s)=\frac{12}{k_{F}^{3} s} \int k \frac{\sin (k s)}{1+\exp \left[\left(\hbar^{2} / 2 m k_{B} T\right)\left(k^{2}-\tilde{k}^{2}\right)\right]} d k .
$$

where $\tilde{k}$ is implicitly specified by the condition $\rho_{T}(0)=\left(2 / 3 \pi^{2}\right) k_{F}{ }^{3}$. For low temperatures and small $s$, Eq. (7.10) may be expanded in the usual way ${ }^{37}$ :

$$
\rho_{T}(s) \approx \rho\left[\frac{3 j_{1}\left(k_{F} s\right)}{k_{F} s}+\frac{\pi^{2}}{2 k_{F}^{4}}\left(\frac{m k_{B} T}{\hbar^{2}}\right)^{2} \cos \left(k_{F} s\right)\right] .
$$


Comparison of the correction term proportional to $\cos \left(k_{F} s\right)$ with the zero temperature result indicates that finite temperature slightly decreases the range of nonlocality. This decrease will be evident in the subsequent discussion of Fig. 21.

In the one-dimensional case, we have been unable to formulate an analogous thermal average for frozen transverse wave functions. The basic problem is most obvious in a finite box, in which case we begin with a finite set of wave functions $\phi_{n}(z)$ each of which is associated with a particular set of transverse wave functions. At finite temperature the occupation should be fragmented over an infinite set of wave functions $\phi_{n}(z)$. However, we have found no artifice analogous to our previous variation of the $z$ dimension of the box for mapping the finite set of wave functions onto an infinite set.

The final salient effect of one-dimensional geometry is the degree to which it limits the definition of single-particle orbitals. In general, if one diagonalizes the density matrix, one is free to take any independent linear combination of the eigenvectors as the single-particle basis, and no single definition is physically preferred. In the one-dimensional case, however, since each $\phi_{n}(z)$ is irrevocably associated with a particular set of transverse wave functions, it may only be transformed among other wave functions with the same transverse components. In the case of symmetric collisions there are only two $\phi_{n}(z)$ 's for each set of transverse wave functions, and for a general asymmetric collision the $\phi_{n}(z)$ are, in fact, unique. In discussing single-particle effects in collisions, we will capitalize on the uniqueness of the single-particle wave functions $\phi_{n}(z)$. However, it is important to bear in mind that this simplification is peculiar to our restrictive one-dimensional geometry.

\section{Single - particle effects}

Density fluctuations play a significant role in the phenomenology of slab collisions we have described. At virtually all energies, a conspicuous lump appears at the slab edge after the initial compression (cf. Figs. 9 and 14 (a) for example). In low energy collisions, conspicuous sloshing of the density is evident in the final states, and at high energies fluctuations in the density precede the condensation of individual reaction fragments. It is important to consider, therefore, the origin of such fluctuations. One possibility is that they arise as Benard-like instabilities in a theory which is dominated by fluid dynamics. An alternative view is that they are essentially quantum mechanical in origin and reflect single-particle degrees of freedom in nuclear dynamics as fundamentally as shell effects display single-particle behavior in static properties. In the context of our one-dimensional slab geometry, there is strong evidence that the latter view is correct, and that single-particle effects play an essential role in the dynamics.

We will therefore view the dynamics in terms of a set of single-particle wave functions evolving in time in a common self-consistent field. Although in symmetric collisions we could always consider arbitrary linear combinations of the two states originating from corresponding orbitals in the two fragments, it will be useful for our purposes to distinguish the orbitals which originated on each side. We could, in any event, make the distinction precise by introducing a slight asymmetry in the thicknesses of the original slabs and thus in the transverse wave function occupations.

The self-consistent potential is quite smooth, since it includes the convolution of the instantaneous density with a Yukawa force. According to the TDHF equation, each single-particle wave function moves independently in this smoothly varying well. The Pauli principle has been built in from the beginning and is satisfied at all times simply because all the particles are moving in the same potential. Hence, all our intuition concerning the transmission, reflection, and spreading of wave packets in simple smooth wells is directly applicable to TDHF dynamics.

To display single-particle effects concretely, the self-consistent potential and individual wave functions are shown in Fig. 19 for the collision of two slabs with $Q_{1}=a_{2}=1.4 \mathrm{fm}^{-2}$ at $E / A=3.5 \mathrm{MeV}$. (The density profiles at the same time intervals for this collision are displayed in Fig. 9.) Each slab originally contains four orbitals. The contributions to the total density $Q_{n}\left|\phi_{n}(z)\right|^{2}$ from the orbitals originating from the first (most bound) and third states of the left slab are followed in time. In addition, at the latest time, the second and fourth orbitals are also shown. By symmetry, the contributions of the orbitals originating in the right slab are just the mirror images of those in Fig. 19.

From the time of initial contact, $t=0.08 \times 10^{-21}$ sec, until scission begins, $t=0.48 \times 10^{-21} \mathrm{sec}$, the single-particle well has an essentially constant depth of $-50 \mathrm{MeV}$. The single-particle wave functions originating at the left may be decomposed into Fourier components, the dominant velocities being

$$
v_{n}=v_{i} \pm\left[\frac{2\left(e_{n}-W\right)}{m}\right]^{1 / 2},
$$

where $v_{i}$ is the initial velocity of the slab, $e_{n}$ is the single particle eigenvalue of $\phi_{n}$ in the static slab, and $W$ is the well depth. In Fig. 19 it is observed that $\phi_{3}$ quickly crosses the potential well, 


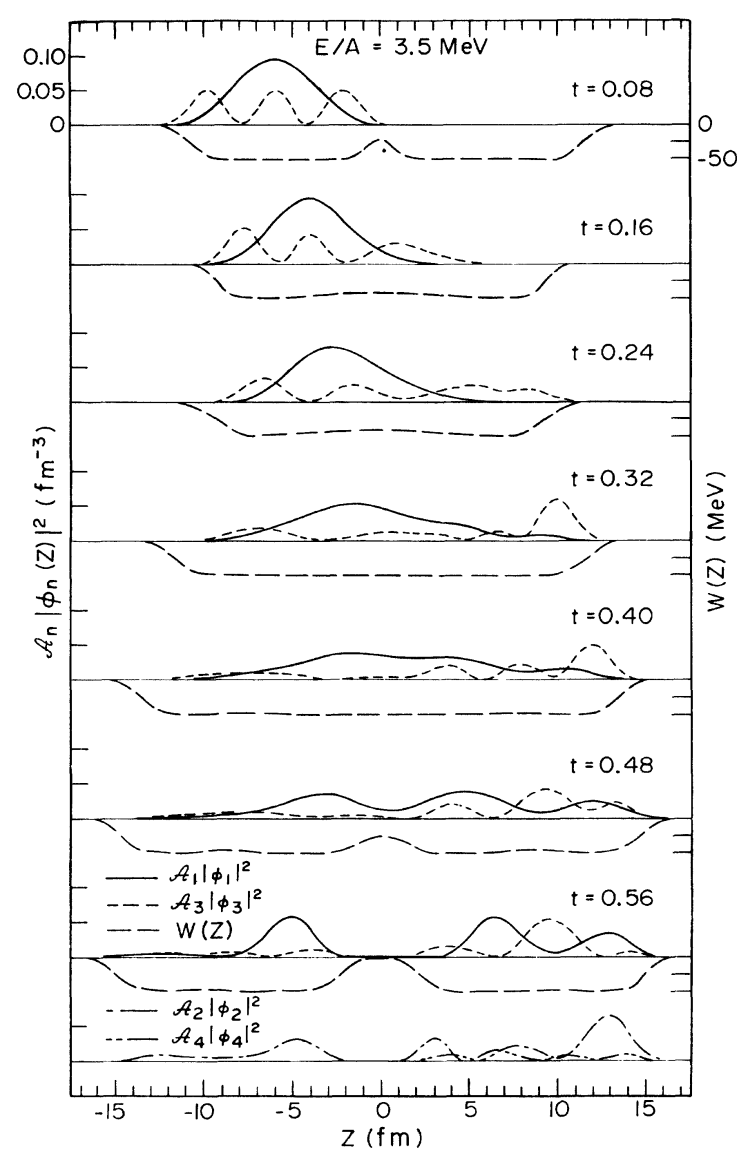

FIG. 19. Contributions of individual single-particle orbitals to the density at various times for the $a_{1}=a_{2}$ $=1.4 \mathrm{fm}^{-2}$ system at $E / A=3.5 \mathrm{MeV}$. In the seven upper graphs, the solid and short-dashed lines denote the contributions of the lowest and third lowest orbitals originating in the left-hand slab. The contributions of the second and fourth orbitals originating in this left slab are shown at $t=0.56$ in the lowest graph. In all cases, the long-dashed curves denote the one-body potential $W(z, t)$.

with most of its probability concentrated near the right edge at time $t=0.32 \times 10^{-21} \mathrm{sec}$. This single orbital accounts for the conspicuous lump in the density at $t=0.32 \times 10^{-21} \mathrm{sec}$ shown in Fig. 9 and is the primary reason the self-consistent potential begins to move back toward the right. This same behavior has been verified at other energies. $A$ priori, we might have expected the highest orbital $\phi_{4}$ to perform the role of striking the right edge of the slab first, creating a bulge in the density and extending the single-particle potential back toward the right. However, the transverse wave function weighting $Q_{4}$ is sufficiently small compared with $Q_{3}$, that although $\phi_{4}$ does reach the edge first, it is ineffective in producing significant observable effects.
The time scale for the first oscillation observed in Fig. 8 for low energy collisions is explained quantitatively by the transit time of $\phi_{3}$. The slab thickness $L=Q / \rho_{0}$ is $9.65 \mathrm{fm}$ for $Q=1.4 \mathrm{fm}^{-2}$. From the data used to construct Fig. 8 , the low energy limit of the elapsed time while the separation distance is less than $9.65 \mathrm{fm}$ during the first oscillation is $0.18 \times 10^{-21} \mathrm{sec}$. This yields a transit velocity of $53.5 \mathrm{fm} / 10^{-21} \mathrm{sec}$, in excellent agreement with the velocity $v_{3}=52.6 \mathrm{fm} / 10^{-21} \mathrm{sec}$ obtained from Eq. (7.12) with $W=-50 \mathrm{MeV}, e_{3}$ $=-35.5 \mathrm{MeV}$, and $v_{i}=0$. At higher energies, such as the case $E / A=3.5 \mathrm{MeV}$ shown in Fig. 19, quantitative comparison is impossible because of the complicated nonlinear process whereby $\phi_{3}$ changes the shape of the right side of the self-consistent well leading to the eventual stretching of the density. The qualitative importance of $\phi_{3}$, however, is still quite evident.

From the post-scission orbitals shown at $t=0.56$ $\times 10^{-21} \mathrm{sec}$ in Fig. 19, it is evident that most of the wave functions originating in the left slab have gone out in the right slab, with only a minor amount of reflection. Thus, case (e) in Fig. 8 corresponds primarily to single-particle orbitals passing through each other.

The situation is significantly different at lower energy. As an example, we consider the case $E / A$ $=1 \mathrm{MeV}$, curve (b) of Fig. 8, for which the postscission wave functions originating in the left slab are shown in the upper portion of Fig. 20. In this case the left slab wave functions first migrate to the right well, as in the previous case. This yields the first maximum of curve (b) in Fig. 8, but the velocities are sufficiently dispersed that the density is rather constant and scission does not occur. The bulk of the wave functions then are reflected back toward the left well, giving rise to a second minimum in the separation distance. Finally, with most of the orbitals back in the left well, scission occurs. It is interesting to note which orbitals dominate the matter actually transmitted to the right slab. The lowest orbital $\phi_{1}$ did not have sufficient time to be completely reflected, so that a significant fraction of it became trapped on the right after scission. The highest orbital $\phi_{4}$, which contains the highest velocity components, actually completed one full cycle of reflections from the right and left edges before finally becoming trapped in the right well on its second transit.

This low energy example suggests the microscopic origin of the rich structure below $E / A=2 \mathrm{MeV}$ portrayed in Figs. 9 and 10. Evidently, at low energy, there is significant dispersion in the velocities and thus in the positions of the individual orbitals after fusion. It is therefore a very delicate question as to whether scission will occur after 


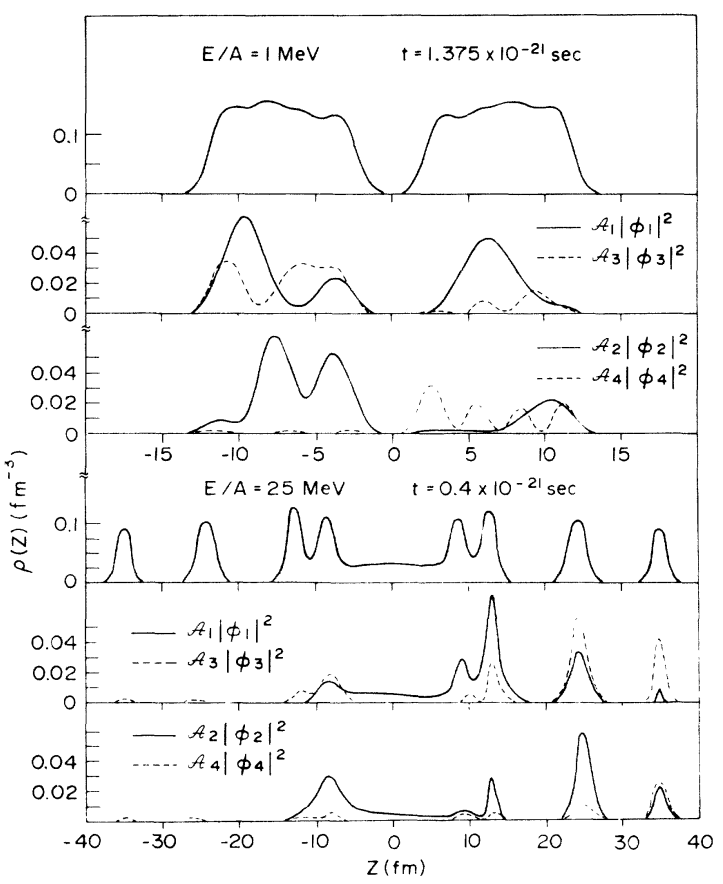

FIG. 20. Contributions of single-particle orbitals at $E / A=1 \mathrm{MeV}$ and $E / A=25 \mathrm{MeV}$ as in Fig. 19. The upper curve for each energy denotes the total density, and the lower curves specify the contributions of each of the four orbitals originating in the left slab.

any given oscillation. This depends in detail upon the positions of the orbitals, ranging from the slowest, which are undergoing their first reflection, to the fastest, which may be on their second or subsequent cycle of reflections. Therefore, minute changes in initial conditions may easily result in qualitative changes in the nature of the reaction.

The single-particle structure of a high energy final state, with $E / A=25 \mathrm{MeV}$, is shown in the lower portion of Fig. 20. In this case, the outermost fragment originates from the familiar lump at the edge of the density caused by $\phi_{3}$ reaching the well edge. As observed in Fig. 20, this fragment is dominated by $\phi_{3}$, but as a result of the nonlinear effects of the self-consistent potential, portions of other single-particle wave functions have also been trapped in the associated potential. Analysis of the other fragments yields a similar conclusion. In each case the fragment appears to be dominated by one single-particle contribution, with subsidiary components arising from all the other states. Thus the following microscopic picture of fragmentation reactions seems to emerge. The slabs pass through each other, with the singleparticle orbitals somewhat dispersed in velocity but continuing predominantly in the forward direc- tion. As the orbitals continue to spread out spatially, density fluctuations begin to emerge when an orbital becomes concentrated in a specific region. This orbital then catalyzes the formation of a reaction fragment, acquiring contributions from other single-particle wave functions via the action of the self-consistent potential.

In the case of our one-dimensional slab geometry, we have presented strong evidence that singleparticle effects are playing an essential role in the reaction dynamics. It remains to be seen whether they remain equally important in more realistic applications. Certainly, with the freedom to modify the definition of single-particle wave functions by performing an arbitrary unitary transformation among the occupied states, the isolation of singleparticle effects will be more difficult. In addition, the question remains whether the addition of transverse degrees of freedom will sufficiently randomize the density matrix that fluid dynamics behavior will be strongly enhanced with a corresponding deemphasis of single-particle properties. ${ }^{38}$

Time - dependent one - body density matrix

Because our many-body wave function is restricted to be a single Slater determinant at all times, all observables are fully specified by the one-body density matrix $\rho\left(\overrightarrow{\mathbf{r}}, \overrightarrow{\mathbf{r}}^{\prime}\right)$ given by Eq. (2.11). The near diagonal region $\overrightarrow{\mathbf{r}} \sim \vec{r}^{\prime}$ is particularly important since only values of $\overrightarrow{\mathbf{r}}-\overrightarrow{\mathrm{r}}^{\prime}$ within the range of the two-body force contribute to the total energy or $\mathrm{HF}$ potential [cf. Eqs. (2.8) and (2.14)]. Furthermore, for large values of $\overrightarrow{\mathbf{r}}-\overrightarrow{\mathbf{r}}^{\prime}$, the density matrix goes to zero because of the random phases of the single-particle wave functions. Because of the importance of the density matrix in the description of the system, a valid approximation to the near-diagonal behavior of this function is crucial to the success of any fluid dynamical reduction of the TDHF equations. ${ }^{38}$ Hence, in this section we briefly examine the density matrix in our TDHF solutions.

Since all of the dynamics of our system pertains to the $z$ coordinate, we restrict our attention to this dependence of $\rho$. It is convenient to remove two trivial factors from the density matrix by defining

$$
\tilde{\rho}(z, s)=e^{-i m v(z) s / \hbar} \rho(z)^{-1} \rho(z+s / 2, z-s / 2),
$$

where $v(z)$, the velocity field, is defined by Eq. (5.2a). The phase factor simply removes a dependence associated with the mean velocity $v(z)$, while the second factor, $\rho(z)^{-1}=\rho(z, z)^{-1}$ normalizes $\tilde{\rho}$ to unity at $s=0$. Thus $\tilde{\rho}$ is the nor malized density matrix as seen in a frame moving with the local 
mean fluid velocity $v(z)$.

The reduced density matrix for the ground state of a three-dimensional Fermi gas at finite temperature may be calculated from Eq. (7.10). Figure 21(a) and 21(b) show the density matrix at various temperatures at saturation density $\rho_{0}$ and at $2 \rho_{0}$. These curves indicate the qualitative effects of a finite temperature. In accord with Eq. (7.6) the off-diagonal range of $\rho$ decreases with increasing density and decreases with increasing temperature at a fixed density. Furthermore, since thermal excitations are effective in a region within $k_{B} T$ of the Fermi surface, for a given temperature, the effect of heating is greater at lower densities. Finally, in addition to the decrease of the off-diagonal range, the negative fluctuation for $s \sim 3 \mathrm{fm}$ in Fig. 21(b) decreases with increasing temperature, consistent with the expectation that the quantal Pauli correlations between particles should diminish in a hot system.

With this background we now turn to the density matrix for the $Q_{1}=Q_{2}=2.4 \mathrm{fm}^{-2}$ system at $E / A$ $=25 \mathrm{MeV}$. Two positions, $z_{1}$ and $z_{2}$, have been selected in the frame of the shock wave as shown in Fig. 18. The real and imaginary parts of $\tilde{\rho}(z, s)$ are shown in Fig. 21(c) at the 3 times displayed in Fig. 18. In a steady state fluid dynamical behavior, the system should be characterized by a time-independent density and temperature at these positions. This is indeed the case at $z=z_{2}$ (not shown), where the curves $\operatorname{Re} \tilde{\rho}\left(z_{2}, s\right)$ at times $t=0.08 \times 10^{-25}$ and $t=0.10 \times 10^{-21} \mathrm{sec}$, are indistinguishable from the curve $\tilde{\rho}\left(k_{F}^{0} s\right)$ and the imaginary parts are essentially zero. That the density matrix in the interior of a ground state slab agrees with the Slater density (7.6) is, of course, expected from the behavior of the static HF solutions. However, the additional information provided by the agreement of $\tilde{\rho}$ with $\tilde{\rho}_{\mathrm{SL}}$ at $z=z_{2}$ is that no significant disturbance of the density matrix has propagated ahead of the transition region shown in Fig. 18.

In contrast to the simple behavior at $z=z_{2}$ in front of the transition region, the behavior of $\tilde{\rho}$ at $z=z_{1}$ behind the shock shows a richer time dependence. The most evident feature of this curve at all times is the shrinking of the $s$ scale with $\rho^{-1}$, reflecting the one-dimensional geometry. For comparison, Fig. 21(d) shows both the one- and three-dimensional results at zero temperature computed from Eqs. (7.9) and (7.6), respectively. Figure 21(c) is in much better agreement with the one-dimensional scale. Also note that while the large $s$ behavior of Re $\rho$ changes significantly with time, the small $s$ behavior, in particular the curvature at $s=0$ and the zero crossing at $s \sim 1.6 \mathrm{fm}$, is remarkably time independent. With increasing time the pronounced negative fluctuation damps strongly and the range increases slightly, so that by the time $t=0.12 \times 10^{-21} \mathrm{sec} \tilde{\rho}$ agrees rather well with the one-dimensional curve of Fig. 21(d).
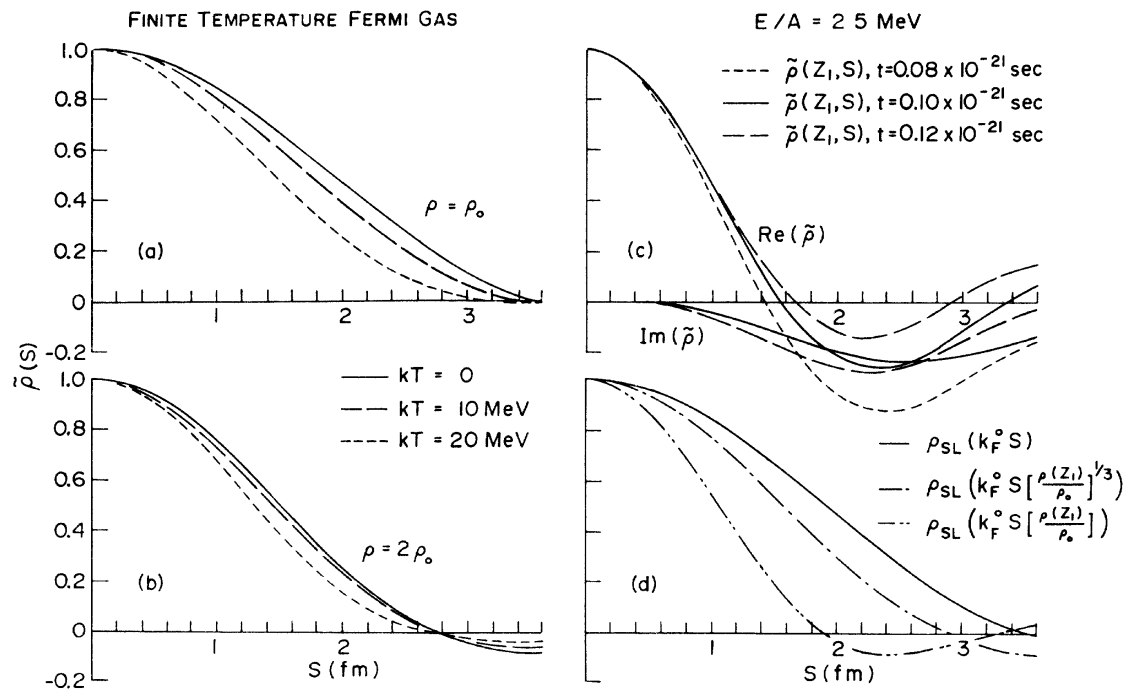

FIG. 21. Normalized one-body density matrices defined according to Eq. (4.13) as a function of relative coordinate $s$. Graph (a) displays the density matrix of a three-dimensional Fermi gas at nuclear density $\rho_{0}$ at three temperatures. Part (b) shows the analogous results at $2 \rho_{0}$. Graph (c) displays the real and imaginary parts of the density matrix for the $Q_{1}=a_{2}=2.4 \mathrm{fm}^{-2}$ system at the position $z_{1}$ denoted in Fig. 18 for 3 times. For comparison, part (d) shows the zero temperature Fermi gas results for nuclear density (solid curve), for the local density at $z_{1}$ in the three-dimensional case (dash-dot curve), and for the local density at $z_{1}$ in the one-dimensional case (dash-dot-dot curve). 
This transient behavior appears to be a nonequilibrium phenomena, so that finite temperature approximations to large $s$ behavior of $\tilde{\rho}$ become questionable. Indeed, an equilibrium finite temperature density matrix has a smaller negative fluctuation than the Slater result, whereas the initial nonequilibrium result has a much larger fluctuation. Furthermore, finite temperature effects seen in Fig. 21(b) are much smaller than the transients in Fig. 21(c). Part of this is undoubtably due to the smaller phase space available for excitation in the one-dimensional result, although the transients seem too large to be due to this alone. One final complication arises from the fact that $\tilde{\rho}\left(z_{1}, s\right)$ has a significant imaginary component at all times except $t=0.08 \times 10^{-21} \mathrm{sec}$ when it must be real by symmetry. The complexity of $\tilde{\rho}$ indicates a velocity dispersion ${ }^{38}$ among the various wave functions $\phi_{n}$ and renders interpretation in terms of thermalized density matrices difficult.

Figure 22 displays analogous results for a collision with $E / A=2.5 \mathrm{MeV}$. In this case it is most convenient to display the results at a fixed position $z_{3}$ in the center of mass frame. The density distribution has been graphed to indicate the progress of the reaction at each time. At each time $\operatorname{Re} \tilde{\rho}\left(z_{3}, s\right)$ is compared with the one-dimensional zero-temperature Slater result. As in the $E / A$

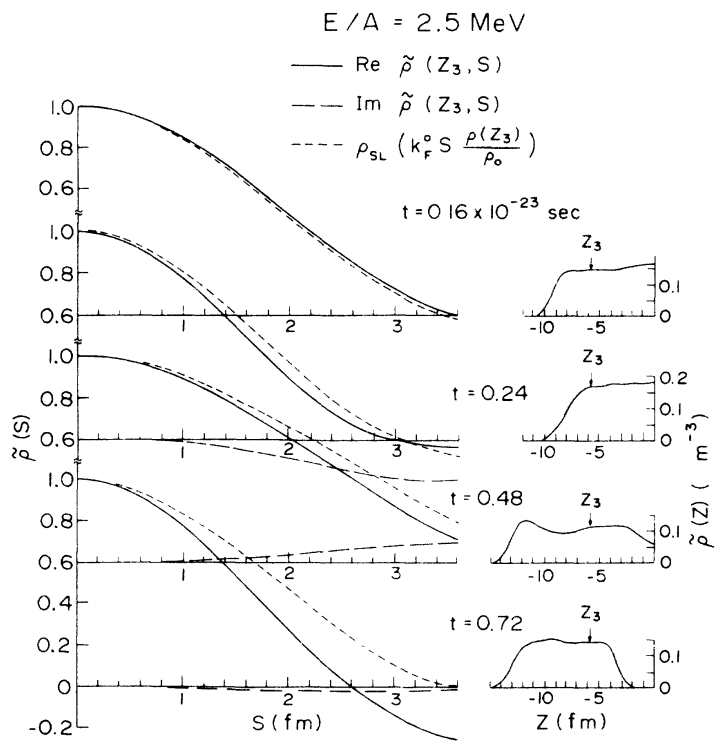

FIG. 22. Normalized one-body density matrices as in Fig. 21 for $E / A=2.5 \mathrm{MeV}$. The left-hand plots present the one-dimensional Fermi gas results (short-dashed curves) and real and imaginary parts of the calculated density matrices (solid and long-dashed curves) at the position $z_{3}$ denoted on the density distributions graphed on the right.
$=25 \mathrm{MeV}$ case, before the interaction region reaches $z_{3}$, these two functions agree. However, at later times, the curvature of $\tilde{\rho}$ at $s=0$ is greater than that of $\tilde{\rho}_{\mathrm{SL}}$ in qualitative agreement with the higher energy case and with the expected behavior at finite temperatures. Although the negative fluctuation appears to be damped at intermediate times $\left(t=0.24 \times 10^{-21}\right.$ and $\left.0.48 \times 10^{-21} \mathrm{sec}\right)$, it is strongly enhanced after scission $(t=0.72$ $\left.\times 10^{-21} \mathrm{sec}\right)$. Finally, the significant imaginary components of $\tilde{\rho}$ render comparison with equilibrium finite temperature results difficult.

In conclusion, aside from the gross scale being determined by the simple one-dimensional Fermi gas result Eq. (7.9) and from the increasing curvature at $s=0$ in excited regions, the detailed structure of the TDHF density matrix is rather complicated. It does not appear to correspond to the structure expected in a simple equilibrium thermodynamic approximation which will probably make the connection of TDHF and fluid dynamics a difficult task. However, since the near-diagonal behavior of the density matrix plays an essential role, it certainly merits further detailed investigation.

\section{DISCUSSION}

As we have seen, the TDHF approach provides a unified description of a broad spectrum of dynamical phenomena encountered in large nuclear systems. This range of phenomena described by the theory is of particular significance, since the evolution of the system is determined only by the two-body interaction and the choice of initial conditions. There is therefore no latitude for an arbitrary selection of collective coordinates or ad hoc parametrization of dynamical processes (e.g., dissipation). When viewed in this light, the results we have presented are indeed encouraging and certainly motivate more realistic calculations.

In addition to the promise of future calculations, several limitations of the TDHF approximation have become evident during the course of this work and therefore require discussion. Some of these problems are specific to the one-dimensional geometry we have considered and will disappear, or at least be minimized, in calculations of higher dimensionality. Others, however, are fundamental to the TDHF approximation and their consideration is therefore relevant for more realistic calculations. Unfortunately, we offer no resolution of these fundamental difficulties here, but simply attempt to provide some insight into the limitations of TDHF.

Those problems specific to one dimension arise from the omission of significant degrees of free- 
dom in our slab geometry. The decoupling of transverse degrees of freedom implicit in our calculations has already been alluded to several times. Recall that the dependence of the singleparticle wave functions upon the transverse coordinates is time-independent, and so irrelevant to the dynamical calculation. However, had these degrees of freedom not been frozen in, the slab symmetry might have broken down as a result of instabilities associated with clustering in the transverse directions. The infinite and homogeneous slab solution we have used must be, by symmetry, an energetically stationary configuration of the system. Should transverse instabilities exist, this configuration would represent an unstable equilibrium which could not be sustained in the presence of fluctuations. In this case it follows that our dynamical calculations have generated mathematically correct though physically implausible solutions to the TDHF equations because of the constraint to configurations of unstable equilibrium.

In addition to the problem of the existence of unstable solutions, the neglect of degrees of freedom is also expected to have severe implications concerning estimates of dissipation derived from our TDHF calculations. For low energy excitations of the physical system, for which there is sufficient time for effective coupling between available modes, energy will flow into the omitted modes, leading to a dissipation higher than the TDHF result. This effect may be seen, for example, in the persistence of oscillations in our calculations of single slab dynamics in Sec. V (cf. Fig. 4). Here the paucity of modes resulted in the apparent excitation of only two or three modes, giving rise to a Poincaré time so short that the results may hardly be characterized as damped. On the other hand, for high energy - short time collisions, the energy loss to neglected degrees of freedom might be expected to be smaller, and therefore the results of a restricted TDHF calculation in this regime may be more valid. It is encouraging to note that, as shown in Fig. 12, more than $90 \%$ of the bombarding energy is dissipated in collisions of a few $\mathrm{MeV}$ per nucleon, a phenomenon strongly suggestive of the experimentally observed dissipation in strongly damped collisions. ${ }^{39}$

Finally, it is important to note that the one-dimensional geometry has eliminated all features associated with the finite transverse extent of real nuclei. Since only compressional modes are present in one dimension, our calculations have omitted shape oscillations which comprise the dominant collective behavior in low energy nuclear physics. Similarly, in head-on collisions of real nuclei, the buildup of density is significantly limited by the possibility of matter splashing out in the transverse directions. Of course, the presence of elastic or nearly elastic peripheral collisions is also excluded in one dimension, so that our calculations do not include this essential feature of many heavy ion reactions.

From the above discussion it is clear that the solutions to the TDHF equations will undergo essential qualitative changes when calculations are extended to two dimensions, and that most of the difficulties we have discussed so far will vanish. In contrast, the extension from two to three dimensions appears to be much less crucial, as no essential new qualitative effects are expected to appear. It is therefore hoped that many quantitative effects in real three-dimensional nuclei can be mocked up in suitable axially symmetric twodimensional calculations. ${ }^{40}$ Indeed, considering the magnitude of the numerical difficulties involved, the realization of this hope is essential if TDHF is to become a practical quantitative tool for nuclear physics.

We now consider those problems which are intrinsic to the TDHF approximation, independent of dimensionality. We have already seen an example of the general property of symmetry conservation in the case of the slab solutions being in unstable equilibrium with respect to transverse perturbations. If the TDHF determinant initially possesses a symmetry conserved by the resultant $\mathrm{HF}$ Hamiltonian, such as translational invariance in the transverse directions, then that symmetry will be a property of the system at all later times. This symmetry conservation is a phenomenon familiar from static $\mathrm{HF}$ calculations and arises in a variety of dynamical situations. For example, it forbids final states involving mass transfer for reactions initiated by identical target-projectile combinations and prohibits triaxial excitations for systems initially axially symmetric. One possible way of circumventing the unphysical restrictions imposed by symmetry conservation while still retaining a mean-field approximation is to treat the residual two-body interaction in the BCS approximation, as discussed below.

Another fundamental point raised by our calculations concerns the interpretation of the final states in TDHF. Under appropriate conditions TDHF describes the approximate evolution of a wave packet of solutions to the full many-body problem. ${ }^{11}$ One might therefore hope to follow the decomposition of the wave packet into asymptotic channel states in the limit as $t \rightarrow \infty$, thereby extracting reaction amplitudes from TDHF. However, even if projection onto all the relevant reaction channels were practical, the nonlinear nature of the TDHF equations allows portions of the initial wave packet in different final channels to 
interact with one another, even asymptotically, so that the whole notion of a scattering amplitude becomes questionable.

As an example of this problem, consider a case in which the real quantal system has nonvanishing reaction amplitudes in several distinct final channels, each having a different shape, mass distribution, and relative velocity. In the TDHF approximation the components of the wave function in these different channels may become trapped in a common potential well freely interacting and exchanging momentum, thereby giving rise to a single fragment localized in coordinate space. One plausible interpretation might be that each fragment which emerges should be dominated by a single Slater determinant, or equivalently, that the matrix quantity $\rho^{2}-\rho$ be small for each fragment considered separately. In the case of slab geometry an equivalent statement is that the metric in the space spanned by the $\phi$ 's, $L$, be a projector when each fragment is considered separately. This was indeed the case for the $E / A=3.5 \mathrm{MeV}$ reac tion of the $Q_{1}=Q_{2}=1.4 \mathrm{fm}^{-2}$ system, for which the quantity $\operatorname{Tr}\left(L-L^{2}\right) / \operatorname{Tr} L$ was $3.5 \%$ when evaluated for each fragment. However, note that for the barrier penetration reaction of Fig. 6 , this quantity is $+17 \%$ for the transmitted fragment.

An alternative approach to the interpretation of the TDHF final states is to insist that the TDHF wave function be used only to specify average few body properties of the final state and that the TDHF approximation itself is not designed to give matrix elements of many-body operators like scattering amplitudes correctly. This type of approach is consistent with the one-body nature of the TDHF approximation and has the intuitive appeal that a Slater determinant which evolves so as to continually maximize its overlap with the true wave function should at least be capable of describing mean values of few-body operators. As an example of the kind of information which may be extracted in this way, we briefly consider the case of a symmetric collision. By virture of symmetry conservation, symmetric reactions result in final states for which the density $\rho(z)$ is an even function of $z$ for a coordinate system in which the center of mass is at the origin. For initial conditions leading to two-fragment final states, reactions involving mass transfer are then apparently forbidden when the wave function is examined at the superficial level of the one-body density. Recalling that $\rho(z)$ is an average property (expectation value of a one-body operator) for the TDHF wave function, this result is perfectly sensible, for reactions leading to asymmetric final states are always present with equal amounts of left and right asymmetry, so that the average property is always symmetric. (Note that in the cases we have considered, this symmetry is not unstable. Since our numerical calculations did not enforce left-right symmetry, perturbations due to numerical noise would have resulted in asymmetric solutions had the system been unstable.) However, an average measure of the mass transfer present in the final state may straightforwardly be obtained. ${ }^{41}$ Let us define a one-body operator $\hat{N}^{R}$, which is the number operator for particles on the right-hand side of the $x-y$ plane:

$$
\begin{aligned}
\hat{N}^{R} & =\int \theta(z) \hat{\psi}^{\dagger}(\overrightarrow{\mathrm{r}}) \psi(\overrightarrow{\mathrm{r}}) d^{3} r \\
& \equiv \int \hat{\psi}^{\dagger}(\overrightarrow{\mathbf{r}}) N^{R}\left(\overrightarrow{\mathrm{r}}, \overrightarrow{\mathbf{r}}^{\prime}\right) \hat{\psi}\left(\overrightarrow{\mathbf{r}}^{\prime}\right) d^{3} r d^{3} r^{\prime},
\end{aligned}
$$

where $\hat{\psi}(\overrightarrow{\mathbf{r}})\left(\hat{\psi}^{\dagger}(\overrightarrow{\mathbf{r}})\right)$ is the annihilation (creation) operator for particles at the point $\vec{r}$. Then, by definition

$$
N^{R}\left(\overrightarrow{\mathbf{r}}, \overrightarrow{\mathbf{r}}^{\prime}\right)=\delta\left(\overrightarrow{\mathbf{r}}-\overrightarrow{\mathbf{r}}^{\prime}\right) \theta(z) .
$$

The quantity

$$
\Sigma^{R} \equiv\left(\left\langle\left(\hat{N}^{R}\right)^{2}\right\rangle-\left\langle\hat{N}^{R}\right\rangle^{2}\right)^{1 / 2} /\left\langle\hat{N}^{R}\right\rangle,
$$

where the expectation value is to be taken in the TDHF state at $t \rightarrow+\infty$, is a time-independent measure of the fractional mass dispersion in the reaction. Using the fact that $N\left(\overrightarrow{\mathbf{r}}, \overrightarrow{\mathbf{r}}^{\prime}\right)$ is a projector, $\Sigma^{R}$ may be rewritten in terms of the density matrix of the right fragment $\rho^{R}$ as

$$
\Sigma^{R}=\left[\operatorname{Tr} \rho^{R}-\operatorname{Tr}\left(\rho^{R}\right)^{2}\right]^{1 / 2} / \operatorname{Tr} \rho^{R}
$$

with

$$
\rho^{R}\left(\overrightarrow{\mathbf{r}}, \overrightarrow{\mathbf{r}}^{\prime}\right)=\theta(z)\left[\sum_{i \text { occupied }} \psi_{i}(\overrightarrow{\mathbf{r}}) \psi_{i}^{*}\left(\overrightarrow{\mathbf{r}}^{\prime}\right)\right] \theta\left(z^{\prime}\right) .
$$

The appearance of the quantity $\operatorname{Tr}\left[\rho^{R}-\left(\rho^{R}\right)^{2}\right]$ in Eq. (8.4) is not unexpected, for while the full ma$\operatorname{trix} \rho^{R}-\left(\rho^{R}\right)^{2}$ specifies the total departure from a single determinant including admixtures of the same as well as different particle number, the diagonal elements of this matrix specify only the dispersion in particle number. As $t \rightarrow-\infty$, when the total wave function may be decomposed into two spatially separated determinants, $\Sigma^{R}$ is zero, corresponding to the definite (infinite in slab geometry) number of nucleons on the right. At later times, however, $\Sigma^{R}$ need not be zero. Unfortunately, while $\Sigma^{R}$ is a sensible quantity for finite systems, for slabs $\Sigma^{R} \sim \Omega^{-1 / 2}$, and so is always zero. This result is reasonable because each function $\phi_{n}(z, t)$ is coupled to a number of plane waves proportional to $\Omega$. The nucleons in these plane waves all have the same (independent) probability for being found on the right, the precise value depending upon the details of $\phi_{n}$. Therefore, 
the resultant mass distribution is binomial and has a fractional variance porportional to $\Omega^{-1 / 2}$.

From the above discussion it is clear that it is very difficult to extract anything other than the most gross reaction information from the TDHF solutions. However, one type of approach in which the TDHF solutions would be used as the zeroth order approximation in a more general theory which would yield such information is as follows. Let us consider a finite set of eigenfunctions of the static HF potential at time $t=0$. Using the same initial conditions for the unoccupied wave functions as for the occupied ones, we may evolve all wave functions according to Eq. (2.9), with the HF potential determined only by the occupied orbitals. Such a calculation then generates a set of time-dependent orbitals which can serve as a basis for either a coupled channels or generator coordinate calculation. The former has the attractive feature that the entrance channel is coupled to other channels only via two-particle-two-hole processes, while the latter has an advantage over conventional generator-coordinate calculations in that the collective variable is selected by the system rather than by ansatz.

A final fundamental difficulty in the TDHF approximation is the neglect of the residual two-body interaction. In addition to the new qualitative feature of breaking symmetry conservation, the residual force will certainly have an as yet unassessed impact on dynamics. At high energies, for example, one might ask to what extent the residual interaction affects the velocity distribution in the transition region of a shock wave. Certainly much of the effect of the two-body force is included in the scattering from the step in the mean field induced by the density change at the interface. How ever, additional scattering from the residual interaction also occurs in reality, and it remains a quantitative question whether it plays a significant role in determining the thickness and structure of the shock transition region.

Although the coupled channels approach described above treats the residual interaction exactly in principle, it is sufficiently difficult in terms of computation so as to make a more tractable approximation attractive. Such an approach, very similar in spirit to TDHF, is the time-dependent Hartree-Fock Bogoliubov (TDHFB) approximation. Following the notation of Valatin, ${ }^{42}$ the dynamical equations may be expressed in terms of matrices of twice the dimension of the density matrix. In addition to the density matrix $\rho$ and HF potential $W$, the system is specified by the pairing field and pairing potential matrices

$$
\chi\left(\overrightarrow{\mathbf{r}}, \overrightarrow{\mathbf{r}}^{\prime}\right)=\left\langle\hat{\psi}(\overrightarrow{\mathbf{r}}) \hat{\psi}\left(\overrightarrow{\mathbf{r}}^{\prime}\right)\right\rangle,
$$

$$
U\left(\overrightarrow{\mathrm{r}}, \overrightarrow{\mathrm{r}}^{\prime}\right)=\frac{1}{2} \iint \hat{V}\left(\overrightarrow{\mathrm{r}}, \overrightarrow{\mathbf{r}}^{\prime} ; \overrightarrow{\mathrm{r}}^{\prime \prime}, \overrightarrow{\mathrm{r}}^{\prime \prime \prime}\right) \chi\left(\overrightarrow{\mathrm{r}}^{\prime \prime}, \overrightarrow{\mathbf{r}}^{\prime \prime \prime}\right) d \overrightarrow{\mathrm{r}}^{\prime \prime} d \overrightarrow{\mathrm{r}}^{\prime \prime \prime} .
$$

Of course, the expectation value in Eq. (8.6a) is in a time-dependent BCS state of indefinite particle number. Defining the augmented matrices

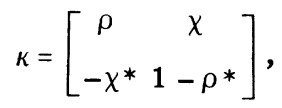

$$
\begin{aligned}
& M=\left[\begin{array}{cc}
h-\lambda & U \\
-U^{*} & -(h-\lambda)^{*}
\end{array}\right],
\end{aligned}
$$

where the HF Hamiltonian $h$ is given by Eq. (2.6) and the chemical potential $\lambda$ specifies the particle number, the equation of motion analogous to Eq. (2.10) is

$$
i \hbar \dot{\kappa}=[M, \kappa]
$$

Although $\kappa^{2}=\kappa$ (in analogy with $\rho^{2}=\rho$ for TDHF), $\operatorname{Tr} \kappa$ is infinite, so that we cannot simply evolve a finite number of eigenfunctions of $\kappa$, as is the case in TDHF. However, the numerical solution of the matrix equations (8.8) may not be completely out of the question, and would begin to introduce some of the significant effects of the residual interaction into the dynamical problem in the same way as pairing theory introduces them into the static problem.

In conclusion, it is evident that substantive and challenging problems remain in the application of TDHF to physical situations. However, the modest successes of the one-dimensional calculations we have presented here certainly motivate continued effort on both the computational and conceptual aspects of the theory. Hopefully the remaining problems will prove sufficiently tractable so that TDHF will indeed fulfill the intended role of providing a foundation for a microscopic theory of nuclear dynamics.

\section{ACKNOWLEDGMENTS}

It is a pleasure to acknowledge the many productive discussions we have had with A. Kerman, M. Baranger, and F. Villars during the course of this work. Additional thanks are also due J. Griffen, G. Bertsch, C. Pethick, and P. J. Siemens for stimulating interactions, and to N. Glendenning for his role in organizing the 1975 Berkeley Sum mer Workshop at which many useful discussions took place. 


\section{APPENDIX A: NUMERICAL SOLUTION OF THE STATIC HF EQUATIONS}

\section{Static iteration procedure}

For a given number of particles $Q$ per $\mathrm{fm}^{2}$, the set of equations (4.11), (4.16), (4.20), (4.21), (4.22), and (4.23) is solved self-consistently. By symmetry, the eigenfunctions $\phi_{n}(z)$ are alternately even and odd functions, and therefore only require solution in one-half of the slab.

The Schrödinger equation (4.16) is solved directly in coordinate space, since expansion in an orthonormal basis such as harmonic oscillator functions is ill-suited for the variety of slab shapes encounted in the time-dependent problem. The Numerov ${ }^{43}$ method is used with a mesh size of $\Delta z$ $=0.2 \mathrm{fm}$ and the boundary conditions that $\phi_{n}$ or $\phi_{n}^{\prime}$ vanish at the origin and that $\phi_{n} \sim \exp \left[-z\left(2 m e_{n} /\right.\right.$ $\left.\hbar^{2}\right)^{1 / 2}$ ] at the mesh edge.

The iteration procedure is started from a Fermi density distribution corresponding to a slab thickness of $\boldsymbol{Q} / \rho_{0}$ and surface thickness $2.1 \mathrm{fm}$, nor malized to the total mass $Q$. Given a density $\rho^{i}(z)$, where $i$ denotes the iteration number, the singleparticle field $W^{i}(z)$ is calculated from Eq. (4.11) using an integration algorithm for the Yukawa potential described below. The eigenvalues, $e_{n}^{i+1}$, and eigenfunction $\phi_{n}^{i+1}(z)$ are then calculated.

The number of occupied orbitals $N^{i+1}$ and the Fermi energy $\epsilon_{F}^{i+1}$ are determined by solving the equation

$$
Q=\sum_{n=1}^{\infty} \theta\left(\epsilon_{F}^{i+1}-e_{n}^{i+1}\right) \frac{2 m}{\hbar^{2}}\left(\epsilon_{F}^{i+1}-e_{n}^{i+1}\right)
$$

Given $\epsilon_{F}^{i+1}$, the weighting factors $Q_{n}^{i+1}$ and the new density $\rho^{i+1}$ are straightforwardly constructed.

The convergence of the iterative solution for the density is improved by using the average of the two most recent solutions

$$
\tilde{\rho}^{i+1}=\frac{1}{2}\left[\rho^{i}+\rho^{i+1}\right],
$$

to construct the HF potential $W^{i+1}$.

Twenty iterations provide adequate convergence for the slabs under consideration. For the case of $Q=1.4 \mathrm{fm}^{-2}$, the precision is specified by

$$
\max \left(\frac{\left|\rho^{20}(z)-\rho^{19}(z)\right|}{\rho^{20}(z)}\right) \sim 5 \times 10^{-4},
$$

and

$$
\sum_{n=1}^{N}\left(\frac{\left|e_{n}^{20}-e_{n}^{19}\right|}{e_{n}^{20}}\right) \sim 10^{-4} .
$$

Convolution of the force with the density

After integration of the Yukawa potential over transverse coordinates, the following integral must be evaluated at the mesh points $z_{j}=j \Delta z$.

$$
U_{j}=U\left(z_{j}\right)=\int_{-\infty}^{\infty} e^{-1 z_{j}-z^{\prime !} / a} \rho\left(z^{\prime}\right) d z^{\prime} .
$$

Because the mesh size $\Delta z=0.2 \mathrm{fm}$ is not sufficiently small relative to the range of the force, Simpson's rule is inadequate. An alternative algorithm suited to this exponential integral may be derived by writing

$$
U_{j}=\sum_{n} \int_{z_{n}-\frac{1}{2} \Delta z}^{z_{n}+\frac{1}{2} \Delta z} e^{-z_{j}-z^{\prime} \mid / a} \hat{\rho}_{n}\left(z^{\prime}\right) d z^{\prime},
$$

where $\hat{\rho}_{n}$ is the second order finite difference approximation to $\rho$ over the interval $\left(z_{n}-\frac{1}{2} \Delta z, z_{n}\right.$ $\left.+\frac{1}{2} \Delta z\right)$ :

$$
\begin{aligned}
\hat{\rho}_{n}\left(z^{\prime}\right)= & \rho_{n}+\left(z^{\prime}-z_{n}\right)\left(\frac{\rho_{n+1}-\rho_{n-1}}{2 \Delta z}\right) \\
& +\frac{\left(z^{\prime}-z_{n}\right)^{2}}{2} \frac{\rho_{n+1}-2 \rho_{n}+\rho_{n-1}}{(\Delta z)^{2}}
\end{aligned}
$$

Equation (A5) is written with the notation $\rho_{n}=\rho\left(z_{n}\right)$. Integrating Eq. (A4) and rearranging terms yields the result

$$
U_{j}=\sum_{q \geqslant 0} X_{q}\left(\rho_{j+q}+\rho_{j-q}\right),
$$

where

$$
\begin{aligned}
& X_{q \geqslant 2}=a e^{-q Y}\left(B_{0}+e^{Y} B_{+}+e^{-Y} B_{-}\right) \\
& X_{q=1}=a e^{-Y}\left(B_{0}+e^{Y} B_{+}+e^{-Y} B_{-}-\left(Q+B_{+}\right) e^{Y}\right), \\
& X_{q=0}=2 a\left(Q+e^{-Y} B_{-}+1-e^{-Y / 2}\right), \\
& B_{0}=\left(-\frac{4}{Y^{2}}+\frac{3}{2}\right) \sinh \frac{Y}{2}+\frac{2}{Y} \cosh \frac{Y}{2}, \\
& B_{ \pm}=\left(\frac{2}{Y^{2}} \pm \frac{1}{Y}+\frac{1}{4}\right) \sinh \frac{Y}{2}-\left(\frac{1}{Y} \pm \frac{1}{2}\right) \cosh \frac{Y}{2} \\
& Q=\left(\frac{2}{Y^{2}}+\frac{1}{Y}+\frac{1}{4}\right) e^{-Y / 2}-\frac{2}{Y^{2}},
\end{aligned}
$$

and

$$
Y=\frac{\Delta z}{a} .
$$

The method of integration (A6) with $X_{q}$ defined by (A7) is significantly superior to Simpson's rule which corresponds to

$$
\begin{aligned}
& X_{q>1}=\Delta z e^{-q Y}, \\
& X_{q=0}=\frac{\Delta z}{a} .
\end{aligned}
$$

Since the constants $X_{q}$ in Eq. (A6) need be calculated only once and then stored, this algorithm requires no more computation than Simpson's rule, both for the static and time-dependent problem. 
For our applications, truncation of the summation in Eq. (A6) at $q=25$ results in adequate precision.

\section{APPENDIX B: NUMERICAL SOLUTION OF THE TDHF EQUATIONS}

Equation (4.29) presents the problem of solving $N$ coupled equations of the form

$$
i \hbar \frac{\partial \phi}{\partial t}(z, t)=h[\rho(t)] \phi(z, t) .
$$

We define a mesh of step size $\Delta z$ in the $z$ coordinate and of step size $\Delta t$ in the time coordinate. The wave functions have value $\phi_{j}^{n}$ at the points $z_{j}=j \Delta z, t_{n}=n \Delta t$. The spatial second derivative appearing in $h$ is approximated by the usual threepoint finite difference expression, while the time derivative is approximated by a two-point formula, so that Eq. (B1) becomes

$$
i \hbar\left(\frac{\phi_{j}^{n+1}-\phi_{j}^{n}}{\Delta t}\right)=\sum_{k} h_{j k}^{n+1 / 2} \phi_{k}^{n+1 / 2} .
$$

We define the Hamiltonian matrix $h_{j k}^{n+1 / 2}$ as

$h_{j k}^{n+1 / 2}=-\frac{\hbar^{2}}{2 m(\Delta z)^{2}}\left(\delta_{j k+1}+\delta_{j k-1}-2 \delta_{j k}\right)+W_{j}^{n+1 / 2} \delta_{j k}$,

where $W_{j}^{n+1 / 2}$ is the value of the HF potential at the point $\left(j, n+\frac{1}{2}\right)$. Note that $h$ is tridiagonal, the only nonzero elements occurring on or adjacent to the diagonal.

The right-hand side of Eq. (B2) requires knowledge of $h \phi$ at the half points in the time mesh. The wave function at such times is unknown, so that $(h \phi)^{n+1 / 2}$ must be approximated. For the moment we ignore the time dependence of $h$ and discuss the implications of various approximations for $\phi^{n+1 / 2}$.

The most naive approximation is to replace $\phi^{n+1 / 2}$ by $\phi^{n}$, so that, in an obvious matrix notation

$$
i \hbar \frac{\phi^{n+1}-\phi^{n}}{\Delta t}=h \phi^{n}
$$

or, solving for $\phi^{n+1}$,

$$
\phi^{n+1}=\left(1-i \frac{\Delta t}{\hbar} h\right) \phi^{n} .
$$

Equation (B5) is explicit in the sense that the new wave function $\phi^{n+1}$ is given directly in terms of the old one $\phi^{n}$. However, while Eq. (B5) is conceptually simple, it can be shown to lead to serious numerical difficulties. In particular, as $h$ is Hermitian, the eigenvalues of $1-i h \Delta t / \hbar$ have modulus greater than or equal to 1 . Therefore, time evolution by continued application of Eq. (B5) results in exponential amplification of that component of $\phi$ associated with the eigenvalue of largest modulus. As the original Eq. (B1) was unitary, such amplification is unphysical and therefore undesirable.

The instability associated with the explicit method may be eliminated by approximating $\phi^{n+1 / 2}$ by the average of $\phi^{n}$ and $\phi^{n+1}$, thus

$$
i \hbar \frac{\phi^{n+1}-\phi^{n}}{\Delta t}=\frac{1}{2} h\left(\phi^{n}+\phi^{n+1}\right) \text {. }
$$

Equation (B6) is an implicit approximation to Eq. (B1) in the sense that the set of simultaneous equations

$$
\left(1+i \frac{h \Delta t}{2 \hbar}\right) \phi^{n+1}=\left(1-i \frac{h \Delta t}{2 \hbar}\right) \phi^{n}
$$

must be solved for the unknowns $\phi^{n+1}$. Note that Eq. (B7) has the formal solution

$$
\phi^{n+1}=\left(\frac{1-i h \Delta t / 2 \hbar}{1+i h \Delta t / 2 \hbar}\right) \phi^{n} .
$$

The time evolution operator in Eq. (B8) is manifestly unitary, leading to no unphysical amplification as all its eigenvalues have unit modulus. In addition, as can be verified by series expansion, for a time-independent Hamiltonian the algorithm defined by Eq. (B8) is accurate through $\theta\left(\Delta t^{2}\right)$. Indeed, Eq. (B8) is nothing but the $[1,1]$ Padé approximant to $e^{-i \hbar \Delta t / \hbar}$, also known as the CrankNicholson or Caley approximation.

In practice, the solution of $\mathrm{Eq}$. (B7) requires the inversion of the tridiagonal matrix $1+i h \Delta t / 2 \hbar$. This inversion has been accomplished by a very stable and efficient version of Gaussian elimination, which has often been described in the literature. ${ }^{44}$ The boundary conditions imposed upon $\phi$ are that the wave function vanish at the ends of the coordinate mesh, far outside the interaction region.

The above algorithm assumes the Hamiltonian $h$ to be time-independent. In the case of the TDHF equations, it is simply generalized to

$$
\phi^{n+1}=\left(\frac{1-i h^{n+1 / 2} \Delta t / 2 \hbar}{1+i h^{n+1 / 2} \Delta t / 2 \hbar}\right) \phi^{n} .
$$

We have found the following scheme adequate for approximating $h^{n+1 / 2}$. We first solve for the wave functions $\bar{\phi}$, defined by the approximation $h^{n+1 / 2}$ $\sim h^{n}$, i.e.,

$$
\bar{\phi}=\left(\frac{1-i h^{n} \Delta t / 2 \hbar}{1+i h^{n} \Delta t / 2 \hbar}\right) \phi^{n} .
$$

With the wave functions $\bar{\phi}$, we construct the Hamiltonian $\bar{h}$. The approximation to $h^{n+1 / 2}$ used in Eq. (B9) is then

$$
h^{n+1 / 2} \sim\left(h^{n}+\bar{h}\right) / 2 .
$$


Thus, we must solve the system of simultaneous equations twice for each step in time. However, the extra effort is well justified as the approximation $h^{n+1 / 2} \sim h^{n}$ in Eq. (B9) results in a continual decrease in the energy of the system, because of the use of a somewhat retarded potential. The approximation $h^{n+1 / 2} \sim \bar{h}$ correspondingly results in an increasing energy, because of the use of an advanced potential. An alternative to (B11) is to first calculate wave functions $\bar{\phi}$ at the half-time step $n+\frac{1}{2}$ by replacing $\Delta t$ in Eq. (B10) by $\Delta t / 2$ and then use the resulting $h^{n+1 / 2}$ constructed from these wave functions in Eq. (B9). Although this method requires the same computing time as the use of Eq. (B11) and yields comparable accuracy for the small time steps used in our calculations, when the time step is doubled it yields an absolute error in energy conservation which is roughly half as large as with the former algorithm. In calculations with 2 or more dimensions, some effort should be expended to find a way to approximate $h^{n+1 / 2}$ from the knowledge of $h$ at the previous time steps.

We have found a step in $z, \Delta z$, of $0.2 \mathrm{fm}$ adequate for all calculations. A time step

$$
\Delta t \lesssim \frac{\Delta z}{4 v_{\max }}
$$

proves sufficient, where $v_{\max }$ is the largest speed involved in the initial conditions. Decreasing $\Delta z$ and $\Delta t$ results in no significant improvement. A typical calculation involves between 150 and 300 points in $z$ and 500 to 1000 steps in time.

Checks of the accuracy are provided by Gallilean invariance and the conservation laws. A static HF solution remains unchanged in time, apart from a well defined time-dependent phase, and such a system, if given an initial velocity, translates with constant speed. For the collision problem the metric is time-independent with in $\pm 10^{-6}$ and the total energy is conserved within $\pm .05 \mathrm{MeV} / \mathrm{fm}^{2}$. The time independence of the metric simply reflects the fact that Eq. (B 7) is unitary, whereas the energy conservation gives a direct indication of the noise propagated by the integration algorithm.

Another severe test is provided by the time-reversal invariance property of the equations. Integrating from $t_{0}$ to $t_{0}+N \Delta t$, replacing all the $\phi$ 's by their complex conjugates and integrating again for another $N$-time interval, the system should return precisely to the state it occupied at time $t_{0}$. We performed such a test for the collision case $Q_{1}=Q_{2}=1.4 \mathrm{fm}^{-2}$ at $E / A=3.5 \mathrm{MeV}$ integrating 400 time steps from $t=0$ to $t=1 \times 10^{-21} \mathrm{sec}$ and then returning backward. The curve $d(t)$ describing the reverse process is virtually indistinguishable from the direct process plotted in Fig. 6. Similarly, the densities $\rho\left(t_{0}\right)$ and $\rho\left(t_{0}+2 N \Delta t\right)$ agree to within a few percent.

Two other integration methods were also investigated and are briefly described here. One stable alternative to the implicit algorithm (B7) is the explicit three-point approximation

$$
\begin{aligned}
\frac{i \hbar}{2 \Delta t}\left(\phi_{j}^{n+1}-\phi_{j}^{n-1}\right)= & -\frac{\hbar^{2}}{2 m \Delta z^{2}}\left(\phi_{j+1}^{n}+\phi_{j-1}^{n}\right) \\
& +\left(\frac{W_{j}^{n}}{2}+\frac{\hbar^{2}}{2 m \Delta z^{2}}\right)\left(\phi_{j}^{n-1}+\phi_{j}^{n+1}\right) .
\end{aligned}
$$

Although this method suffers from the drawback that it is not unitary, it offers the advantage that $\phi_{j}^{n+1}$ may be evaluated explicitly in terms of known quantities without solving a system of simultaneous equations. For comparable accuracy in collision problems, Eq. (B13) requires a time step at least 8 times smaller than Eq. (B7). The explicit and implicit computing times differ by a factor much smaller than 8, however, since the explicit method does not require the intermediate calculation of $\rho^{n+1 / 2}$ or the solution of simultaneous equations required by the implicit method. The real motivation for investigating the algorithm (B13) is the eventual consideration of two-dimensional problems for which the solution of the simultaneous equations for the implicit method is much more cumbersome. From these 1-dimensional results, it appears that such explicit methods merit serious consideration in subsequent two-dimensional applications.

A second alternative is to use an unstable algorithm but to maintain sufficient precision that the noise level does not become significant before all physically relevant information is obtained. This can be achieved, for example, by increasing the precision with which the time derivative is evaluated. Using the Adams-Moulton predictor corrector method with Zonnefeld's starting formulas, ${ }^{45}, 46$ a five point formula having a precision through terms in $(\Delta t)^{4}$ was used to integrate Eqs. (4.29) with the approximation (B3) for $h$. However, sensible results required so small a time step that computation time became prohibitive. 
* This work supported in part through funds provided by the U.S. Energy Research and Development Administration under Contract No. AT(11-1)-3069.

$\dagger$ Present address: CEN Saclay, Gif-sur-Yvette, France.

\$Present address: California Institute of Technology, Pasadena, California.

$\S$ Alfred P. Sloan Foundation Research Fellow.

${ }^{1}$ P. A. M. Dirac, Proc. Cambridge Phil. Soc. 26, 376 (1930).

${ }^{2}$ J. W. Negele, Phys. Rev. C $\underline{1}, 1260$ (1970).

${ }^{3}$ X. Campi and D. W. L. Sprung, Nucl. Phys. A194, 401 (1972).

${ }^{4}$ D. Vautherin and D. M. Brink, Phys. Rev. C $\underline{5}, 626$ (1972); D. Vautherin, Phys. Rev. C 7, 6 (1973).

${ }^{5} \mathrm{M}$. Beiner, H. Flocard, Nguyen Van Giai, and P. Quentin, Nucl. Phys. A238, 29 (1975).

${ }^{6}$ G. F. Bertsch and S. F. Tsai, Phys. Rep. 18, 125 (1975).

${ }^{7}$ T. DeGrand, R. Jaffe, K. Johnson, and J. Kiskis, Phys. Rev. (to be published).

${ }^{8} \mathrm{G}$. E. Brown, Unified Theory of Nuclear Models and Forces (North-Holland, Amsterdam, 1967).

${ }^{9} \mathrm{~F}$. Villars, in Rendiconti della Schuola Internazionale di Fisica "Enrico Fermi," XXIII Corso (Academic, New York, 1963); in Dynamical Structure of Nuclear States, Proceedings of the 1971 Mont Tremblant International Summer School, edited by D. J. Rowe (Univ. of Toronto Press, Toronto, 1972).

${ }^{10}$ L. Schafer, Nucl. Phys. A194, 497 (1972).

${ }^{11}$ A. K. Kerman and S. E. Koonin (unpublished).

${ }^{12}$ T. H. R. Skyrme, Phil. Mag. 1, 1043 (1956); Nucl. Phys. 9, 615 (1959).

${ }^{13} \mathrm{~J}$. W. Negele, in Effective Interactions and Operators in Nuclei, Lecture Notes in Physics 40, edited by B. R. Barrett (Springer, Berlin, 1975).

${ }^{14} \mathrm{~J}$. W. Negele and D. Vautherin, Phys. Rev. C $\underline{5}, 1472$ (1972); 11, 1031 (1975).

${ }^{15}$ Y. M. Engel, D. M. Brink, S. J. Krieger, and D. Vautherin, Nucl. Phys. A249, 215 (1975).

${ }^{16} \mathrm{E}$. DePassos, Ph.D. thesis, Massachusetts Institute of Technology, 1974 (unpublished).

${ }^{17} \mathrm{C}$. Y. Wong, J. A. Maruhn, and T. Welton (private communication).

${ }^{18}$ W. D. Myers and W. J. Swiatecki, Ann. Phys. (N.Y.) 55, 395 (1969).

${ }^{19} \bar{M}$. A. Thorpe and D. J. Thouless, Nucl. Phys. A156, 255 (1970); P. Bonche, ibid. A191, 609 (1972).

${ }^{20} \mathrm{~J}$. J. Griffin and K. K. Kan, in Proceedings of the Third International Symposium on the Physics and Chemistry of Fission, Rochester, 1973 (International Atomic Energy Agency, Vienna, 1974), paper SM$174 / 58$.

${ }^{21}$ H. Flocard, P. Quentin, A. K. Kerman, and D. Vautherin, Nucl. Phys. A203, 433 (1973).
${ }^{22}$ S. E. Koonin (unpublished); A. J. Sierk and J. R. Nix in Proceedings of the Third International Symposium on the Physics and Chemistry of Fission, Rochester, 1973 (see Ref. 20), paper SM-174/74.

${ }^{23}$ J. R. Nix and A. J. Sierk, Phys. Scripta 10A, 94 (1974).

${ }^{24}$ H. Feshbach, A. K. Kerman, and S. E. Koonin (unpublished).

${ }^{25}$ W. Busza, in High Energy Physics and Nuclear Structure, 1975, AIP conference proceedings No. 26, edited by D. E. Nagle (unpublished).

${ }^{26}$ C. Y. Wong and T. A. Welton, Phys. Lett. $\underline{49 B}, 243$ (1974).

${ }^{27}$ S. E. Koonin, Ph.D. thesis, Massachusetts Institute of Technology, 1975 (unpublished).

${ }^{28}$ M. I. Sobel, P. J. Siemens, J. P. Bondorf, and H. A. Bethe, Nucl. Phys. A251, 502 (1975).

${ }^{29}$ G. F. Bertsch, Phys. Rev. Lett. 34, 697 (1975).

${ }^{30}$ S. E. Koonin (unpublished).

${ }^{31}$ A. A. Amsden, G. F. Bertsch, F. H. Harlow, and J. R. Nix, Phys. Rev. Lett. 35, 905 (1975).

${ }^{32}$ G. F. Chapne, J. F. Johnson, E. Teller, and M. S. Weiss, Phys. Rev. D 8, 4302 (1973).

${ }^{33}$ W. Sheid, H. Müller, and W. Greiner, Phys. Rev. Lett. 32,71 (1974).

${ }^{34}$ A. E. Glassgold, W. Heckrotte, and K. W. Watson, Ann. Phys. (N.Y.) 6, 1 (1959).

${ }^{35}$ G. E. Brown, Many Body Problems (North-Holland, Amsterdam, 1972); J. Goldstone and K. Gottfried, Nuovo Cimento X13, 849 (1959).

${ }^{36}$ S. O. Bäckman, A. J. Jackson, and J. Speth, Phys. Lett. 56B, 209 (1975)

${ }^{37}$ L. D. Landau and E. M. Lifshitz, Statistical Physics (Pergamon, New York, 1958).

${ }^{38}$ C. Y. Wong, J. A. Maruhn, and T. A. Welton, Nucl. Phys. A253, 469 (1975).

${ }^{39}$ F. Hanappe, Phys. Rev. Lett. 32, 738 (1974).

${ }^{40} \mathrm{~S}$. E. Koonin, in Proceedings of the Fourth Conference on Gross Properties of Nuclei and Nuclear Excitations, Hirschegg, Austria, 1976 (unpublished).

${ }^{41} \mathrm{~J}$. Brandt and I. Kelson, Phys. Rev. 183, 1025 (1969).

${ }^{42} \mathrm{~J}$. G. Valatin, in Lectures in Theoretical Physics, edited by W. E. Brittin (Interscience, New York, 1962), Vol. IV.

${ }^{43}$ C. E. Fröberg, Introduction to Numerical Analysis (Addison-Wesley, Reading, 1965).

${ }^{44}$ R. S. Varga, Matrix Iterative Analysis (Prentice-Hall, Englewood Cliffs, 1962); R. D. Richtmeyer and K. W. Morton, Difference Methods for Initial. Value Problems (Wiley, New York, 1967), 2nd ed.

${ }^{45}$ L. P. Meissner, Lawrence Berkeley Computer Center Program No. D2 BKY ZAM, 1965 (unpublished).

${ }^{46} \mathrm{~J}$. A. Zonnefeld, Automatic Numerical Integration, Mathematical Center Tract No. 8 (Mathematisch Centrum, Amsterdam, 1964), p. 23. 